\title{
Cultural intelligence predicts intercultural negotiation effectiveness
}

\author{
By
}

Yee-Wei Ooi

Thesis submitted in fulfilment of the requirements for the degree of Masters of Science in CrossCultural Psychology

March 2014

Victoria University of Wellington

Te Whare Wananga o te Upoko o te Ika a Maui

Aotearoa/New Zealand 


\begin{abstract}
The current research project aims to replicate Imai and Gelfand's (2010) study examining cultural intelligence (CQ) as a predictor of intercultural negotiation effectiveness in an experimental setting. Research on culture and negotiation to date has mainly focused on comparing negotiation processes and styles within mono-cultural teams across cultural boundaries rather than examining them in intercultural settings (with negotiators from different cultures interacting with each other). There is now evidence suggesting that negotiators behave differently in intra- and intercultural negotiation settings. Furthermore, outcomes for intercultural negotiations are generally suboptimal compared to intracultural negotiations. Correlational findings by Imai and Gelfand (2010) suggested that CQ functions as a predictor of intercultural negotiation outcomes. We hypothesise that CQ, an individual's ability to adapt and perform effectively in intercultural situations, can predict intercultural negotiation effectiveness beyond individual characteristics that have been shown to predict intracultural negotiation effectiveness (i.e., cognitive ability, emotional intelligence, and the Big 5 personality traits). We experimentally manipulated group compositions to examine the effect of CQ on intercultural negotiation. Findings support the hypothesis that cultural intelligence predicts intercultural negotiation effectiveness, while controlling for cognitive ability, emotional intelligence, and personality. Examining the importance of individual CQ dimensions, we found overall CQ to drive intercultural negotiation outcomes rather than any one particular factor. Theoretical and applications implications are discussed.
\end{abstract}

Keywords:

Cultural intelligence, intercultural negotiation, negotiation effectiveness 


\section{Acknowledgements}

I would like to take this opportunity to thank all the people who have supported me throughout this journey. I would like to thank my supervisor, Dr. Ronald Fischer, for his guidance and advice. More importantly, his enthusiasm and positive outlook has been an inspiration. Thank you also to Anna Lee for our joint data collection efforts. Thanks to the members of the Centre for Applied Crosscultural Research for their encouragement and feedback. I would like to thank our three interns, Dana Cary, Lauren Richardson, and Isobel Cairns, for their time and support. Thanks also to all the participants who took part in both stages of the study. Last but not least, heartfelt thanks to my parents for their advice and support, and for being with me every step of this journey. 


\section{Table of contents}

Introduction....................................................... 1

Culture and Negotiation......................................... 2

Negotiation tactics and sequences............................... 5

Cultural intelligence.......................................... 6

Cultural intelligence and intercultural negotiation................... 9

Outline of study and hypotheses............................... 12

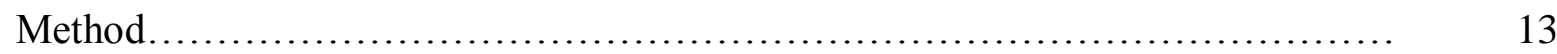

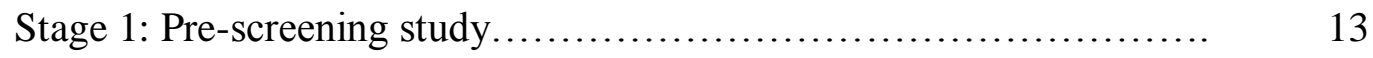

Participants.............................................. 13

Procedure.................................................. 14

Measures................................................. 15

Stage 1 results.............................................. 16

Reliability analysis of CQ scale............................. 16

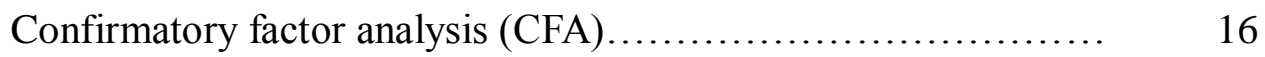

Inferential statistics..................................... 17

Stage 2: Experiment......................................... 18

Participants........................................... 19

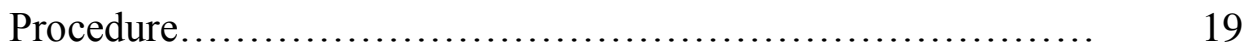

Negotiation task ...................................... 19

Pre-negotiation survey.................................. 20

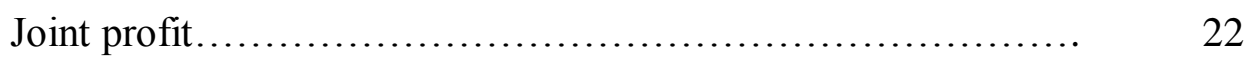

Post-negotiation survey................................. 22

Coding negotiation tactics............................... 23

Coding integrative negotiation sequences................... 23

Design............................................. 24 
Results.................................................................. 24

Descriptive statistics........................................ 24

Manipulation check............................................ 26

Tests of hypotheses........................................... 27

Sequences of integrative information negotiation................... 29

Discussion........................................................... $\quad 30$

Theoretical implications....................................... 34

Practical applications.......................................... 36

Limitations, strengths, and future research........................ 39

Conclusion.................................................. 41

References........................................................... 42

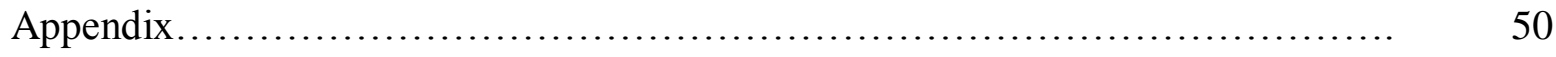




\section{List of Tables}

Table 1 Descriptive statistics and alphas of overall CQ and facet-level CQ (Stage 1: Pre-

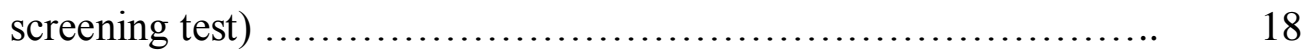

Table 2 Means and standard deviations of group joint profit................. 26

Table 3 Summary of group main effect while controlling for individual difference

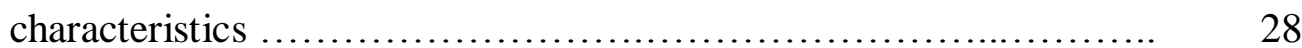

Table 4 Summary of integrative negotiation sequences and joint profit of dyads.

\section{List of Figures}

Figure $1 \quad$ Bar graph presenting mean joint profits. 


\section{Cultural intelligence predicts intercultural negotiation effectiveness}

Advances in technology in the past couple of decades have transformed the way humans interact with one another. With increased interaction across borders come increased opportunities for cultural misunderstandings, tensions, and conflicts (Ang, Van Dyne, \& Tan, 2011). The current research aims to replicate Imai and Gelfand's (2010) study on cultural intelligence (CQ) as a predictor of intercultural negotiation effectiveness. Cultural intelligence is defined as an individual's capability to adapt and perform effectively in intercultural situations (Earley \& Ang, 2003). Negotiation is the process whereby two or more parties discuss common and conflicting interests in order to reach an agreement of mutual benefit (Harris \& Moran, 1996). Although there has been research investigating the predictors of negotiation effectiveness, the majority of studies have been conducted in mono-cultural settings (e.g., Barry \& Friedman, 1998; Nauta \& Sanders, 2000; Ma \& Jaeger, 2005, 2010; Fulmer \& Barry, 2004; Elfenbein, Der Foo, White, Tan, \& Aik, 2007; Cullen, Muros, Rasch, \& Sackett, 2013). Furthermore, current literature on culture and negotiation has remained largely comparative (Adair \& Brett, 2005; Imai \& Gelfand, 2010). Most of the research in the literature to date has focused on comparing negotiation processes and styles within mono-cultural teams across cultural boundaries (intracultural negotiation) rather than examining negotiation processes and outcomes with negotiators from different cultures interacting with each other (intercultural negotiation).

The present research therefore proposes to replicate findings by Imai and Gelfand (2010) who found cultural intelligence as a potential individual difference characteristic that negotiators can be selected and trained in order to achieve optimal intercultural negotiation effectiveness. We also aim to extend Imai and Gelfand's (2010) findings by experimentally manipulating dyad compositions to examine the effect of cultural intelligence on intercultural negotiation. More importantly, we wish to examine whether cultural intelligence has an effect on intercultural negotiation beyond other individual difference characteristics found to predict 
intracultural negotiation effectiveness. Previous research have found the following individual characteristics to predict intracultural negotiation effectiveness: cognitive ability (IQ) (e.g., Barry \& Friedman, 1998; Kurtzberg, 1998; Fulmer \& Barry, 2004), emotional intelligence (EQ) (e.g., Fulmer \& Barry, 2004; Der Foo, Elfenbein, Tan, \& Aik, 2004; Elfenbein et al., 2007), and the Big Five personality traits including extraversion (e.g., Barry \& Friedman, 1998; Nauta \& Sanders, 2000; Cullen et al., 2013), agreeableness (e.g., Barry \& Friedman, 1998; Nauta \& Sanders, 2000), conscientiousness (e.g., Cullen et al., 2013), and openness to experience (e.g., Ma \& Jaeger, 2005, 2010).

Furthermore, the current research hopes to add to the culture and negotiation literature by moving beyond cross-cultural comparisons of negotiation styles to investigating the actual processes people engage in when negotiating in intercultural settings. Gaining a deeper understanding of how individuals with various levels of cultural intelligence interact and the outcomes of such interactions has the potential to not only inform intercultural training programmes aimed at improving effective intercultural communication, but also the recruitment and selection of personnel for overseas assignments or roles which require significant intercultural interactions.

The following sections of the introduction will cover: a) a brief literature review of culture and negotiation, including negotiation tactics and sequences, b) a short introduction to cultural intelligence (CQ) and how it is conceptualised in the current research, and c) an outline of the current study and hypotheses.

\section{Culture and Negotiation}

In most cultures, negotiation is considered as a means of distributing resources (Adair \& Brett, 2004). As such, negotiation outcomes can be either distributive or integrative (Adair \& Brett, 2004). A distributive negotiation outcome reflects only one party's interest (i.e., a win-lose scenario) whereas an integrative negotiation outcome reflects both or all parties' interests (i.e., a win-win scenario) (Adair \& Brett, 2004). As one of the first researchers to compare inter- and intra-cultural 
negotiation outcomes, Adler and Graham (1989) further pointed out that one of the reasons for the dearth of intercultural negotiation research was the assumption that people's behaviour will remain consistent in both intercultural and intracultural (or mono-cultural) negotiation contexts.

Nonetheless, when the assumption that people will exhibit consistent negotiation behaviours in intercultural and intracultural negotiation contexts was put to the test, it was found that individuals behave differently in intercultural negotiation situations compared to intracultural negotiation situations (e.g., Adler \& Graham, 1989; Brett \& Okumura, 1998; Adair, Okumura, \& Brett, 2001; Adair, 2003). For example, Adair and colleagues have found that negotiators from high context cultures (i.e., Japanese negotiators) usually adapt their negotiation behaviours to the more explicit behaviours of negotiators from low context cultures (i.e., American negotiators) (Adair et al., 2001; Adair, 2003). Negotiators from high-context cultures generally employ high-context communication, information that is either in the physical context or internalised in the person with little being communicated in the explicit words or message (Harris \& Moran, 1996). On the other hand, negotiators from low context cultures generally rely on explicit verbal messages (Harris \& Moran, 1996).

Furthermore, studies have shown that even though Japanese negotiators understood their American partners' priorities and adopted similar negotiation behaviour (i.e., Japanese negotiators exhibited more explicit information sharing), joint gains in intercultural negotiation situations were still low compared to intracultural negotiation situations (Adair et al., 2001; Adair, 2003). The researchers speculated that although intercultural negotiators were able to behaviourally adapt in intercultural situations (i.e., Japanese negotiators using more explicit information sharing strategies); they were unable to fully understand their negotiation partner's priorities (Adair et al., 2001). Adair and colleagues (Adair et al., 2001) found that intercultural negotiators used significantly more clarifying statements compared to intracultural negotiators, indicating that intercultural negotiators still have difficulties comprehending the priorities of their negotiation partner (Adair et al., 2001). It therefore appears that, even though intercultural negotiators are aware 
of the preferred negotiation styles of their negotiation counterparts and were able to adapt to their partner's preferred negotiation styles, they were unable to fully comprehend the other party's interests and priorities and were unable to achieve comparable levels of joint gains compared to intracultural negotiation situations.

Another possible explanation for lower joint joints in intercultural compared to intracultural negotiation situations could be that intercultural negotiators lacked the motivation to generate joint gains in the first place (Adair et al., 2001). Previous research indicated that Japanese intercultural negotiators reported relatively accurate understanding of American intercultural negotiators' priorities post-negotiation but American intercultural negotiators did not have a comparable level of understanding their Japanese counterparts' priorities (Brett \& Okumura, 1998). This suggests that only Japanese intercultural negotiators possessed adequate understanding to generate joint gains in intercultural negotiations but they choose not to do so, indicating a lack of motivation to generate joint gains (Adair et al., 2001). Adair and colleagues further argued that these findings are consistent with an in-group/out-group explanation for low motivation to generate joint gains in intercultural negotiation settings. During intercultural negotiations, collectivist negotiators (i.e., Japanese negotiators) were negotiating with an out-group (i.e., American negotiators). The literature on individual-collectivism indicated that collectivist cultures, such as Japan, will negotiate cooperatively with in-group members (i.e., other Japanese negotiators) but competitively with out-group members (i.e., American negotiators) (Triandis, 1989).

To the best of my knowledge, the literature on culture and negotiation thus far has suggested that intercultural negotiation outcomes are usually suboptimal compared to intracultural negotiation outcomes (e.g., Adler \& Graham, 1989; Adair et al., 2001; Adair, 2003). Research on culture and negotiation has also highlighted the fact that negotiators behave differently in intercultural compared to intracultural negotiation settings (e.g., Adler \& Graham, 1989; Brett \& Okumura, 1998). As such, individual characteristics that are advantageous for integrative negotiation effectiveness in a mono-cultural context may not be as effective in predicting 
intercultural negotiation outcomes. To date, individual characteristics that have been found to positively correlate with better mono-cultural negotiation outcomes include personality traits such as extraversion (e.g., Barry \& Friedman, 1998, Nauta \& Sanders, 2000; Cullen et al., 2013 ), agreeableness (e.g., Barry \& Friedman, 1998; Nauta \& Sanders, 2000), conscientiousness (e.g., Cullen et al., 2013) and openness to experience (e.g., Ma \& Jaeger, 2005, 2010), cognitive ability (IQ) (e.g., Barry \& Friedman, 1998; Fulmer \& Barry, 2004; Kurtzberg, 1998), and emotional intelligence (EQ) (e.g., Elfenbein, Der Foo, White, Tan \& Aik, 2007; Der Foo, Elfenbein, Tan \& Aik, 2004; Fulmer \& Barry, 2004). Furthermore, there is currently very little research conducted in the culture and negotiation literature regarding the characteristics negotiators can be selected and trained upon in order to specifically maximise intercultural negotiation effectiveness (Imai \& Gelfand, 2010).

Negotiation tactics and sequences

Within the negotiation literature, negotiation tactics generally fall under two broad conceptual strategies: integrative information and cooperative relationship management (Imai \& Gelfand, 2010). In past cross-cultural research, both direct and indirect ways of providing or eliciting integrative information were coded (e.g., Adair, 2003; Adair \& Brett, 2005; Adair et al., 2001). The direct integrative information negotiation code captures explicit information that helps negotiators identify trade-offs, similar preferences, and other alternatives/opportunities to create joint gains (Adair, 2003). Behaviours that encompass the direct integrative information negotiation code include: a) providing information regarding the relative importance of two or more issues and b) asking for the other negotiator's priorities across two or more issues (Imai \& Gelfand, 2010). The indirect integrative information negotiation code captures implicit information that helps negotiators create joint gain (Adair, 2003). Imai and Gelfand (2010) operationalised indirect integrative information negotiation code as making offers across two or more issues. Lastly, the cooperative relationship management negotiation code captures miscellaneous relationship-focused cooperative comments (Imai \& Gelfand, 2010). 
These negotiation tactics create action-response sets of behaviour which then form sequences of negotiation behaviour (Adair \& Brett, 2004). An integrative negotiation sequence is the behavioural sequence engaged by both negotiators in the dyad to maximise joint profits (Weingart, Prietula, Hyder, \& Genovese, 1999). Imai and Gelfand (2010) examined two types of integrative information behaviour sequences: complementary and reciprocal. A complementary sequence of integrative information behaviour occurs when two negotiation tactics within the sequence are not identical in terms of negotiation tactic but are of the same integrative information strategy. A reciprocal sequence of integrative information behaviours, on the other hand, occurs when the two negotiation tactics forming the sequence are identical. Sequences of cooperative relationship management behaviours occurred when a cooperative, non task-focused comment on the negotiators' relationship was followed by a similar cooperative statement (Imai \& Gelfand, 2010). Examples for sequences of integrative information (reciprocal and complementary) and cooperative relationship management behaviours are provided in the Appendix.

\section{Cultural intelligence}

Situated in the organisational and management literature, the multidimensional concept of cultural intelligence (CQ) was introduced as an individual difference that explains why some individuals are able to perform better in intercultural situations compared to others (Earley \& Ang, 2003). Based on contemporary theories of intelligence (Sternberg, 1986), cultural intelligence is defined as an individual's capability to perform and manage effectively in situations characterised by cultural diversity (Earley \& Ang, 2003). Furthermore, as a capability, CQ is flexible and can be improved through experience, education and training (Ang et al., 2011). Culture in this context is conceptualised as a group of people's worldview (Earley, Ang, \& Tan, 2006) which can encompass multiple social groups whose members share a similar worldview.

In the present study, CQ is conceptualised as an ability to perform effectively in situations characterised by cultural diversity. It is important to note that CQ, as defined in the 
current study, is not cultural competence. Cultural competence is defined as "a set of congruent behaviours, attitudes, policies, and structures that come together in a system or agency or among professionals and enables the system, agency, or professionals to work effectively in cross-cultural situations" (Flaskerud, 2007, p. 121). CQ is thus an individual's ability to demonstrate cultural competence by learning new patterns of behaviour and applying them effectively in the appropriate cultural setting (Earley \& Ang, 2003).

Ang and colleagues further conceptualised cultural intelligence as a set of four capabilities, based on the theory of multiple loci of intelligence (Ang et al., 2011). The four capabilities, or factors/facets, of cultural intelligence are meta-cognitive, cognitive, motivational, and behavioural. Meta-cognitive CQ describes an individual's level of conscious cultural awareness during cross-cultural interactions (Ang \& Van Dyne, 2008). As such, individuals with high metacognitive CQ will consciously question and reflect on their own cultural assumptions during an intercultural encounter. Cognitive CQ refers to an individual's level of cultural knowledge (Ang \& Van Dyne, 2008). This factor reflects an individual's knowledge of norms, practices, and conventions in different cultures that was obtained through educational or personal experiences. Motivational CQ describes an individual's capability to direct attention and energy towards learning about and functioning in situations characterised by cultural diversity (Ang \& Van Dyne, 2008). Motivational CQ is derived from the expectancy-value theory of motivation (Wigfield \& Eccles, 2000). The expectancy-value theory of motivation predicts that the direction and magnitude of energy channelled towards a particular task involves two elements: a) the expectation of successfully accomplishing the task, and b) the value associated with accomplishing the task. Therefore, individuals with high motivational CQ would direct their attention and energy towards intercultural situations based on intrinsic interest (Deci, 1975) and confidence in intercultural effectiveness (Bandura, 2002). And lastly, behavioural CQ describes the extent to which an individual acts in an appropriate manner (both verbally and non-verbally) in intercultural situations (Ang \& Van Dyne, 2008). Behavioural CQ thus reflects an individual's capability to display 
appropriate verbal and non-verbal behaviours when interacting with people from different cultures.

Following the conceptualisation of cultural intelligence, Ang and colleagues conducted a series of studies to develop and validate a 20-item cultural intelligence scale (Van Dyne, Ang, \& Koh, 2008). Results of their studies provided evidence that the scale has a robust and meaningful four factor structure, and is generalisable across different samples, time, and methods (i.e., self vs. observer report) (Van Dyne et al., 2008). Furthermore, the studies also support the discriminant and incremental validity of the CQ scale. The scale has demonstrated discriminant validity compared to cognitive ability, emotional intelligence (see Ward, Fischer, Lam, \& Hall, 2009 for alternative findings), cultural judgement decision making (CJDM), interactional adjustment, and mental wellbeing and incremental validity in predicting cultural adjustment and decision making, adjustment, and mental well-being (Van Dyne et al., 2008).

Since the development of the CQ scale, it has been used in a number of studies examining cultural intelligence and its personality correlates (e.g., Ang, Van Dyne, \& Koh., 2006; Ward \& Fischer, 2008; Ward et al., 2009), its role in predicting cross-border leadership effectiveness (e.g., Rockstuhl, Seiler, Ang, Van Dyne, \& Annen, 2011), adaptive overseas work performance (e.g., Chen, Lin, \& Sawangpattanakul, 2011), cross-cultural adjustment (e.g., Lin, Chen, \& Song, 2012; see also Ward et al., 2009; Ward, Wilson, \& Fischer, 2011 for alternative findings), its relationship with cultural essentialism (Fischer, 2011), and intercultural effectiveness outcomes such as cultural adaptation, task performance, and cultural judgement and decision making (Ang et al., 2007).

As cultural intelligence is a relatively new construct, the majority of research in this area has been focused on expanding the construct's nomological network. The development and validation of the CQ scale has provided researchers with more tools with which to examine the phenomenon of why some individuals are better able to adapt and function effectively in intercultural situations compared to others. However, the empirical literature to date has focused largely on the impact of cultural intelligence at the individual level. To the best of my knowledge, with the exception of the study by Imai and Gelfand (2010), no other study has investigated cultural 
intelligence as a predictor of intercultural negotiation effectiveness at the dyadic level of analysis.

Furthermore, the concept of cultural intelligence as an individual's capability to perform effectively in intercultural situations by definition implies interaction with others (Earley \& Ang, 2003). It is therefore important to move beyond examining the antecedents of cultural intelligence at the individual level to investigating its effects at the dyadic and group level of analysis (Gelfand, Imai, \& Fehr, 2008). For example, how do individuals with different levels of cultural intelligence interact? What are the outcomes of these interactions? Additionally, the majority of research in cultural intelligence to date has been correlational and focused on the ability of the construct to predict effective cross-cultural adjustment and adaptation above and beyond other variables, such as emotional intelligence (e.g., Rockstuhl et al., 2011; Lin et al., 2012), the big five personality model (e.g., Huff, Song, \& Gresch, in press), and international experience (e.g., Templer, Tay, \& Chandrasekar, 2006). Nevertheless, it is important to move beyond these correlational findings to experimentally examine the effects of CQ on intercultural interactions. Furthermore, it has been noted that "little is known regarding the precise mechanisms through which CQ exerts its effects on outcomes" (Gelfand et al., 2008, p. 380). Therefore, gaining a deeper understanding of how individuals with high or low levels of CQ interact with others who also vary in their levels of CQ and the effectiveness of such interactions has the potential to inform intercultural training programmes aimed at improving intercultural communication.

\section{Cultural intelligence and intercultural negotiation}

To the best of my knowledge, Imai and Gelfand's (2010) research reported the first empirical evidence supporting cultural intelligence as a predictor of intercultural negotiation effectiveness. The researchers analysed transcripts of negotiating dyads and found that CQ predicted the extent to which negotiators engaged in sequences of integrative information behaviours, which then predicted joint profits. Correlational findings indicate that CQ provides negotiators with psychological characteristics that are beneficial for engaging in effective 
integrative negotiation processes that could lead to joint profit in an intercultural negotiation setting (Imai \& Gelfand, 2010).

Although the exact processes whereby CQ affects intercultural negotiation outcomes is yet undetermined (Gelfand et al., 2008), Imai and Gelfand (2010) predicted that dyads consisting of individuals with higher cultural intelligence will engage in more effective sequences of integrative information behaviours (both reciprocal and complementary) compared to dyads consisting of individuals with lower cultural intelligence in a mixed-motive negotiation setting. Furthermore, more sequencing of integrative information behaviours will then lead dyads to achieve higher joint profit (i.e., better intercultural negotiation outcomes) (Imai \& Gelfand, 2010). The negotiation task in the study was considered mixed-motive because negotiators were required to both claim and create value (Weingart \& Olekalns, 2004). That is, negotiators were required to try and maximise profits not just for themselves, but also for their counterpart, thus generating integrative solutions. The researchers predicted that, since dyads with higher overall cultural intelligence should also have a greater desire to learn and adapt to the culturally diverse situation and the negotiation problem at hand, they would engage in more sequences of integrative information behaviours in order to understand their counterpart's priorities (Imai \& Gelfand, 2010). Additionally, as dyads with higher overall cultural intelligence are more cooperative, they will also engage in more sequences of cooperative relationship management behaviours than dyads with lower overall cultural intelligence (Imai \& Gelfand, 2010). Given the advantages of engaging in integrative negotiation processes, high cultural intelligence dyads should hence achieve higher joint profits in the negotiation simulation (Imai \& Gelfand, 2010).

The researchers found that overall cultural intelligence at the dyadic level predicted the extent to which negotiators exhibited complementary sequences of integrative information behaviours, but not reciprocal sequences of integrative information behaviours or cooperative relationship management behaviours (Imai \& Gelfand, 2010). Notably, individual differences found to be beneficial for integrative intracultural negotiation such as international experience, 
personality characteristics such as openness to experience and extraversion, cognitive ability, and emotional intelligence did not significantly increase sequencing of integrative information behaviours (Imai \& Gelfand, 2010).

More importantly, examination of dyad composition found that it is the negotiator with lower cultural intelligence within the dyad who determines the level of effective sequencing of integrative information and cooperative relationship management behaviours (Imai \& Gelfand, 2010). The frequency of integrative information and cooperative relationship management negotiation sequences were therefore constrained by the lower CQ negotiator. The authors reasoned that, due to the conjunctive nature of negotiation sequences, the dyad composition effect is logical in that sequencing integrative negotiation behaviours is a joint task that requires the contributions of both negotiators (Imai \& Gelfand, 2010). For example, although the negotiator with high cultural intelligence tries to adopt an integrative negotiation strategy, the dyad's overall negotiation outcome would still suffer if the negotiator with low cultural intelligence does not reciprocate the integrative behaviours (Imai \& Gelfand, 2010).

The dyad composition effect may have highlighted one of the limits of cultural intelligence as a predictor of intercultural negotiation effectiveness (J. Liu, personal communication, September 25, 2012). If the level of integrative negotiation processes were more a function of the lower-CQ negotiator in the dyad, it may suggest that cultural intelligence as a construct requires mutuality in order to realise optimal intercultural negotiation outcomes. That is, both negotiators in a given dyad will need to possess equally high CQ in order to reach optimal intercultural negotiation outcomes.

Furthermore, the dyad composition effect could help explain previous research findings where joints gains in intercultural negotiations were lower compared to mono-cultural negotiations when only one intercultural negotiator was able to discern his/her negotiation partner's priorities (Brett \& Okumura, 1998; Adair et al., 2001). For example, Brett and Okumura (1998) found that Japanese intercultural negotiators reported relatively accurate understanding of American 
intercultural negotiators' priorities post-negotiation. However, American intercultural negotiators did not have a comparable level of understanding of their Japanese counterpart's priorities (Brett \& Okumura, 1998). According to the dyad composition effect, both negotiators were required to have similar levels of understanding in order to engage in effective sequencing of integrative negotiation, which in turn leads to higher joint gains. It could be that a lack of understanding of their counterparts' priorities constrained American negotiators from realising opportunities to generate higher joint gains.

\section{Outline of study and hypotheses}

The current research aims to conduct a more direct test of the dyad composition effect in an experimental setting. The previous study by Imai and Gelfand (2010) employed a correlational design and randomly paired negotiators to form intercultural dyads. As such, it is possible that a dyad with high cultural intelligence may have consisted of one negotiator with extremely high CQ and one negotiator with moderate or low CQ. In this case, the effect of mixed-CQ dyads on intercultural negotiation effectiveness would have been masked. The current study proposed to address this issue by experimentally manipulating dyad compositions by assigning domestic and international students from a New Zealand university into four groups consisting of: high CQ dyads, low CQ dyads, and mixed-CQ dyads. The two groups of mixed-CQ dyads will compose of low CQ domestic - high CQ international student and high CQ domestic - low CQ international student dyads. Two mixed-CQ groups were proposed to examine whether country of origin affects the dyad composition effect found by Imai and Gelfand (2010). That is, will the dyad composition effect be stronger for the low CQ domestic or low CQ international negotiator? Based on Imai and Gelfand's (2010) study and research in the negotiation and culture literature, the present research tested the following hypotheses:

1) Hypothesis 1: High CQ dyads will achieve significantly higher joint profits compared to dyads with at least one low CQ negotiator. 
2) Hypothesis 2: CQ predicts significantly higher joint profits beyond individual characteristics associated with intracultural negotiation effectiveness including cognitive ability (IQ), emotional intelligence (EQ), and the Big 5 personality traits (agreeableness, openness to experience, emotional stability/neuroticism, conscientiousness, and extraversion).

3) Hypothesis 3: High CQ dyads will engage in significantly more integrative information behaviour strategies (reciprocal and complementary) compared to dyads with at least one low CQ negotiator.

4) Hypothesis 4: High CQ dyads will engage in significantly more cooperative relationship management behaviours compared to dyads with at least one low CQ negotiator.

\section{Method}

This research is conducted in two stages. Participants were screened for their CQ scores during Stage 1. Based on their CQ scores, participants were then invited to take part in Stage 2 of the study which examined CQ as a predictor of effective intercultural negotiation strategies and outcomes. Ethics approval for both stages of the research was granted by the School of Psychology Human Ethics Committee under the delegated authority of the Victoria University of Wellington Human Ethics Committee

\section{Stage 1: Pre-screening study}

Stage 1 of this research was designed as a screening test for a follow-up study (Stage 2: Experiment).

\section{Participants}

Participants recruited were students from Victoria University of Wellington, New Zealand. A total of 350 international students (232 females; 112 males; $M_{\text {age }}=22.10, S D_{\text {age }}=5.13$ ) and 564 domestic students (408 females; 156 males; $M_{\text {age }}=18.90, S D_{\text {age }}=2.72$ ) were screened for their CQ scores. Of the international student sample, participants include students from the United 
States, United Kingdom, Germany, Australia, China, Vietnam, Malaysia, the Philippines, Samoa, Indonesia, India, South Africa, South Korea, Fiji, Laos, Cambodia, and others. Countries with more than 10 participants were: United States (45), United Kingdom (43), China (30), Vietnam (18), Malaysia (18), Germany (13), the Philippines (12), Samoa (11), India (10) and Indonesia (10). Although international students in general were sampled, the current study focused on recruiting Asian and Pacific international students for Stage 2 of the study.

\section{Procedure}

Three main recruitment strategies were employed for data collection. Participants were recruited for the pre-screening study in person during student orientations, as part of the Introduction to Psychological Research Programme at Victoria University of Wellington, and flyers around campus.

\section{Direct approach}

Potential participants were approached to fill out the survey during the three-day international student orientation held by Victoria International and the one-day Pacific students' orientation prior to the start of the academic year. Participants were also approached during events organised by the Victoria University of Wellington Postgraduate Students' Association (PGSA) held over two days. Potential participants were approached during these events and invited to fill out a pen-and-paper survey. The survey took approximately 10 to 20 minutes to fill out and included demographic and participant contact details. Participants received candy as a token of appreciation. Participants were further informed that they may be contacted to take part in a follow-up study where they may receive a grocery voucher up to the value of NZD\$20.

\section{Introduction to Psychological Research Programme (IPRP)}

The Introduction to Psychological Research Programme (IPRP) is a mandatory course requirement for students enrolled in an introductory psychology course at Victoria University of 
Wellington. Participants recruited through IPRP were required to complete an identical online survey and contact details were recorded for a follow-up invitation. Participants received IPRP credit for completing the survey.

Flyers

English and Chinese-language flyers advertising the pre-screening study were distributed around the main Kelburn campus and the Pipitea campus. These two campuses were targeted as they are the two biggest campuses of the university. Interested individuals emailed their contact information and those from the target countries (i.e., Asian and Pacific countries) were emailed a link to an online version identical to the IPRP version.

\section{Measure}

Cultural Intelligence $(C Q)$

Cultural intelligence was assessed using the 20-item Cultural Intelligence Scale developed by Van Dyne and colleagues (Van Dyne et al., 2008). The scale's four-factor structure has been confirmed in repeated studies (Van Dyne et al., 2008; Ward et al., 2009). The items were measured on a 1 (strongly disagree) to 7 (strongly agree) Likert scale with higher scores indicating higher levels of CQ. Meta-cognitive CQ is measured with four items. An example item for metacognitive CQ is: "I am conscious of the cultural knowledge I use when interacting with people with different cultural backgrounds". Cognitive CQ is measured with six items. An example item for cognitive CQ is: "I know the arts and crafts of other cultures". Behavioural and motivational facets of CQ were measured with five items each. An example behavioural CQ item is "I change my nonverbal behaviour when a cross-cultural situation requires it" and an example motivational CQ item is "I enjoy living in cultures that are unfamiliar to me". 


\section{Stage 1 Results}

\section{Reliability analysis of CQ scale}

As a measure of reliability, Cronbach's alpha was calculated for the 20-item CQ scale for the international and domestic student sample. The overall CQ scale demonstrated high reliability for both the international and domestic student sample $\left(\alpha_{\text {international }}=.89 ; \alpha_{\text {domestic }}=.89\right)$. The scale also demonstrated high reliability on international and domestic student samples at the facet-level: meta-cognitive CQ $\left(\alpha_{\text {international }}=.81 ; \alpha_{\text {domestic }}=.84\right)$, cognitive CQ $\left(\alpha_{\text {international }}=.83\right.$; $\left.\alpha_{\text {domestic }}=.84\right)$, motivational CQ $\left(\alpha_{\text {international }}=.87 ; \alpha_{\text {domestic }}=.85\right)$, and behavioural CQ $\left(\alpha_{\text {international }}=\right.$ $\left..86 ; \alpha_{\text {domestic }}=.88\right)$. All 20 items in the CQ scale performed well for both international and domestic student samples, with alphas remaining above .80 for each item deleted.

\section{Confirmatory factor analysis (CFA)}

Confirmatory factor analysis (CFA) was used to confirm the factor structure of the 20 CQ items. MPlus (Muthén \& Muthén, 2010) software was used to conduct the CFA. Specifying a model where the metacognitive, cognitive, motivational, and behavioural items load onto their respective facets, the overall model in the combined sample had good fit $\left(\chi^{2}(164)=642.25, p<.001\right.$; $\mathrm{CFI}=.94 ; \mathrm{TLI}=.93 ; \mathrm{RMSEA}=.06 ; \mathrm{SRMR}=.05)$. The fit indices were within the acceptable cutoff criteria suggested by Fischer and Fontaine (2011). We also ran a single-factor model which did not fit as well as the proposed four-factor model $\left(\chi^{2}(170)=3710.06, p<.001 ; \mathrm{CFI}=.52 ; \mathrm{TLI}=.47\right.$; RMSEA=.16; SRMR=.12). In addition, a Chi-square difference test showed that the four-factor model fits significantly better than a single factor model $\left(\chi^{2}(6)=3064.82, p<.001\right)$. More importantly, a model in which the samples were split into domestic and international students and factor loadings and intercepts were constrained to be equal across both groups provided acceptable fit $\left(\chi^{2}(360)=910.66, p<.001 ; \mathrm{CFI}=.93 ; \mathrm{TLI}=.92 ; \mathrm{RMSEA}=.06 ; \mathrm{SRMR}=.06\right)$. We therefore concluded that the CQ scale worked well in both samples. Although the four factor structure fit better than the uni-dimensional structure, we are interested in the overall levels of CQ. We have no 
theoretical reason to expect that one or more of the subscales are more important for selecting our participants. As such, we recruited participants on their overall CQ score.

\section{Inferential Statistics}

Independent samples t-tests were conducted to analyse whether international and domestic participants significantly differed in their CQ scores. Compared to domestic participants, international participants scored significantly higher in overall CQ, $t(799)=4.99, p<.001$. Of the four facets of CQ, international participants scored significantly higher in meta-cognitive $(t(799)=$ 2.33, $p=.020)$, cognitive $(t(799)=5.08, p<.001)$, and motivational CQ $(t(743.09)=5.21, p<.001)$. However, international and domestic participants did not differ significantly in behavioural CQ $(t(799)=1.36, p=.18)$. Table 1 presents the descriptive statistics of CQ (at the overall and facet levels) for the international and domestic student sample respectively. 


\begin{tabular}{|c|c|c|c|c|c|c|}
\hline Variable & Minimum & Maximum & $M$ & $S D$ & Median & $\alpha$ \\
\hline \multicolumn{7}{|c|}{ International student sample $(N=350)$} \\
\hline Overall CQ & 1.00 & 7.00 & 4.85 & .78 & 4.90 & .89 \\
\hline Meta- & 1.00 & 7.00 & 5.23 & .99 & 5.25 & .81 \\
\hline \multicolumn{7}{|l|}{ cognitive CQ } \\
\hline Cognitive CQ & 1.00 & 7.00 & 4.06 & 1.03 & 4.17 & .83 \\
\hline Motivational & 1.00 & 7.00 & 5.51 & 1.03 & 5.60 & .87 \\
\hline \multicolumn{7}{|l|}{ CQ } \\
\hline Behavioural & 1.00 & 7.00 & 4.84 & 1.12 & 5.00 & .86 \\
\hline \multicolumn{7}{|l|}{ CQ } \\
\hline \multicolumn{7}{|c|}{ Domestic student sample $(N=564)$} \\
\hline Overall CQ & 2.25 & 6.75 & 4.62 & .74 & 4.60 & .89 \\
\hline Meta- & 1.00 & 7.00 & 5.06 & .91 & 5.00 & .84 \\
\hline \multicolumn{7}{|l|}{ cognitive CQ } \\
\hline Cognitive CQ & 1.00 & 6.67 & 3.71 & 1.05 & 3.83 & .84 \\
\hline Motivational & 1.00 & 7.00 & 5.19 & 1.05 & 5.20 & .85 \\
\hline \multicolumn{7}{|l|}{ CQ } \\
\hline Behavioural & 1.00 & 7.00 & 4.77 & 1.07 & 4.80 & .88 \\
\hline CQ & & & & & & \\
\hline
\end{tabular}

\section{Stage 2: Experiment}

Following the pre-screening study, selected participants were invited back for a followup study based on their CQ scores. Stage 2 of this research was a conceptual replication and extension of the first study investigating the predictive value of CQ for predicting intercultural negotiation effectiveness (Imai \& Gelfand, 2010). The research aimed to examine the effect of CQ on intercultural negotiation effectiveness in an experimental setting. 


\section{Participants}

Participants were selected from Stage 1 based on their overall CQ scores. A total of 80 participants (22 males and 58 females) from Stage 1 took part in Stage 2 of the study. The mean age of participants was $20.58(\mathrm{SD}=4.13)$. Participants were matched on gender and CQ score and then randomly assigned to one of the four experimental groups.

\section{Procedure}

Participants were seated facing each other across two tables with two laptops placed in front of them. Prior to the start of the session, participants read the information sheet outlining the experiment and were given time to ask the researcher any questions they may have. Once they have read the information sheet and have their questions (if any) answered by the researcher, participants signed two copies of the consent form. Participants kept one copy of the consent form while the other was kept by the researcher. All surveys were administered via Qualtrics, an online data collection tool. Participants first filled out a survey measuring emotional intelligence (EQ), cognitive ability (IQ), and personality traits on the laptops. After that, participants were given time to read their confidential role information. Once both participants were ready, they commenced the negotiation. Negotiations were timed and participants were informed they had 15 minutes to agree on the five issues. Once their negotiation was completed, participants filled out a final agreement form indicating their levels of settlement on for each issue. The negotiations were video recorded.

After the negotiation, participants completed a final a post-negotiation survey. They were then debriefed and thanked. Participants received a supermarket voucher with a value of up to $\$ 20$ as appreciation. Participants were informed to contact the researcher if they had any subsequent questions regarding experiment.

\section{Negotiation task}

Based on Imai and Gelfand's (2010) study, the negotiation task was adapted from the 
Towers Market II Coding Manual for Negotiators' Behaviours which has been used in previous research on integrative negotiations (e.g., Imai \& Gelfand, 2010; Weingart, Olekalns, \& Smith, 2004). Participants were randomly assigned the role of a florist or a grocer. Participants were informed that a successful real estate developer has proposed developing a multi-functional market that includes a speciality grocery store and florist with shared interior décor. However, individual businesses in the market will still have separate areas with which to sell their respective products. Participants were informed that they are seriously considering the shared market but needed to negotiate five unresolved issues with the other store owner prior to committing. The five unresolved issues were: a) hours of operation, b) renovations costs, c) floor space, d) temperature, and e) grand opening date.

The role information sheet also included a payoff schedule which listed the possible levels of settlement on each of the five unresolved issues and the number of points associated with each level of settlement. The associated points indicate the importance of each issue to the negotiator. The structure of the payoff schedule was designed to leave room for integrative potential: grand opening date and renovation costs were integrative issues (i.e., both negotiators have different priorities for the two issues); floor space was a compatibility issue (i.e., the best choice for Negotiator A is also the best choice for Negotiator B); and hours of operation and temperature were distributive issues (i.e., both negotiators have different optimal choices). Please refer to Appendix for the complete role information and payoff schedule.

\section{Pre-negotiation survey}

The following questionnaires were included in the pre-negotiation survey. The survey also included demographic information such as gender, ethnicity, and age. IQ and English Proficiency

As the experiment was conducted with international students whose first language is not English, it was important to have a measure of participants' English language proficiency as a 
control variable. English language proficiency was measured using the Shipley Institute of Living Scale (SILS, Shipley, 1940). The 60-item SILS consists of a vocabulary test and an abstract thinking test. The SILS was designed to assess general intelligence in adults and adolescents and has been used to measure English proficiency and general intellectual ability (e.g., Lun, Fischer, \& Ward, 2010; Tse \& Altarriba, 2012).

\section{Emotional intelligence}

Consistent with Imai and Gelfand's (2010) study, emotional intelligence was measured using Schutte et al.'s (1998) 33-item scale. The measure was based on Salovey and Mayer's (1990, cited in Schutte et al., 1998) model of emotional intelligence. The 33-item scale was measured on a 1 (hardly applicable) to 4-point (completely applicable) Likert scale with participants answering the extent to which an item applies to them. An example item in the scale would be "I know when to speak about my personal problems to others". An example of a reverse-worded item would be "It is difficult for me to understand why people feel the way they do". Reliability analysis confirmed good internal reliability of the EQ scale for the overall sample at Stage $2(\alpha=.87)$.

\section{International Personality Item Pool (IPIP)}

Imai and Gelfand (2010) examined openness to experience and extraversion using Goldberg et al.'s (2006) short version of the International Personality Item Pool (IPIP). This experiment also drew ten items for each of the Big 5 personality characteristic (openness to experience, extraversion, neuroticism/emotional stability, agreeableness, and conscientiousness). The items were measured on a 1 (very inaccurate) to 5 (very accurate) Likert scale with participants answering the extent to which an item accurately describes them. Examples of the items are: "I am the life of the party" (extraversion), "I am interested in people" (agreeableness), "I get chores done right away" (conscientiousness), "I have a vivid imagination" (intellect), and "I am relaxed most of the time" (emotional stability). Examples of items that were reversed-scored are: "I don't like to draw attention to myself" (extraversion), "I insult people" (agreeableness), "I avoid my duties" 
(conscientiousness), "I am not interested in abstract ideas" (intellect), and "I have frequent mood swings" (emotional stability). The five personality subscales exhibited good internal reliability in the current sample $\left(\alpha_{\text {Extraversion }}=.86 ; \alpha_{\text {Conscientiousness }}=.82 ; \alpha_{\text {Agreeableness }}=.81 ; \alpha_{\text {Intellect }}=.78 ;\right.$ and $\left.\alpha_{\text {Emotional Stability }}=.87\right)$.

\section{Joint profit}

Joint profit was measured as the sum of points earned by both negotiators across all five issues in the negotiation task.

\section{Post-negotiation survey}

A post-negotiation survey was administered to measure participants' feelings during the negotiation. The survey was adapted from a previous questionnaire used in Brett and Okumura's study (1998) to rule out alternative explanations. The survey consists of 14 items on two dimensions assessing participants' level of frustration (labelled 'frustration') and perceived cooperation during the negotiation (labelled 'cooperation'). The items were measured on a 1 (strongly disagree) to 7 (strongly agree) Likert scale and participants were required to answer the extent to which they agreed on the items. An example item for the frustration dimension would be "I felt frustrated". An example item for the cooperation dimension would be "the other person was cooperative". Both dimensions demonstrated acceptable reliability $\left(\alpha_{\text {frustration }}=.61 ; \alpha_{\text {cooperation }}=.76\right)$.

The survey also included a shortened three-item social attraction scale adapted from McCroskey and colleagues (McCroskey, McCroskey, \& Richmond, 2007). The items were measured using a 'yes/no' format and assessed whether participants they will be willing to have further interactions with their partner in the future. An example item would be "Can you imagine a friendship with the other person?". The social attraction scale demonstrated acceptable reliability ( $\alpha$ $=.57)$.

The scales in the post-negotiation survey were acceptable for research purpose given 
the small number of items.

\section{Coding negotiation tactics}

The video-taped sessions were transcribed and all verbal behaviour was recorded. Two coders content coded negotiation tactics employed by participants using the Towers Market II negotiation simulation coding adopted by Imai and Gelfand (2010). The average inter-rater agreement was high (84.2\%). Definitions for the negotiation tactics, examples, and strategic grouping of codes are provided in the Appendix. Following Imai and Gelfand's (2010) example, we coded for direct and indirect integrative information behaviours. Specifically, we coded providing information regarding the relative importance of two or more issues and asking for the other negotiator's priorities across two or more issues as direct integrative behaviours. We coded behaviours as issuing offers across two or more issues as indirect integrative information behaviour. Cooperative relationship management behaviours were coded as off-task relationship-focused cooperative comments (Imai \& Gelfand, 2010).

\section{Coding integrative negotiation sequences}

Once all transcripts were content coded for negotiation tactics, the sequences of integrative information behaviours were calculated manually for each dyad transcript. Following Imai and Gelfand's (2010) procedure, sequences of integrative information behaviours were examined in two ways: reciprocal and complementary integrative information sequences. A reciprocal sequence of integrative information behaviours occurs when the two negotiation tactics comprising the sequence were identical. For example, Negotiator A informs Negotiator B the two most important issues for him/her (i.e., "grand opening date and temperature are the two most important issues to me") and Negotiator B reciprocates by informing Negotiator A the two most important issues for him/her (i.e., "well, for me it's renovation costs and hours of operation). A complementary integrative negotiation sequence occurs when the two negotiation tactics were not 
identical but were of the same integrative information strategy. An example of a complementary integrative negotiation sequence is when Negotiator A asks which of the five issues is most important to Negotiator B (i.e., "what's the most important issue for you right now?") and Negotiator B informs Negotiator A of the most important issue to him/her (i.e. "uh...probably renovation costs"). Lastly, a cooperative relationship management sequence occurs when a cooperative, miscellaneous comment by one participant was responded in kind by his/her partner. Refer to Appendix for more examples of integrative information behaviours and cooperative relationship management sequences.

\section{Design}

Participants were randomly assigned into four experimental groups comprising of the following dyads: a) low CQ dyads; b) high CQ dyads; c) low CQ domestic - high CQ international student dyads; and d) high CQ domestic - low CQ international student dyads. Low scorers were participants who scored below the overall median and high scorers were those who scored above the overall median. We attempted to recruit participants who scored on the extreme ends of the CQ scale. Intercultural negotiation effectiveness was measured by the mean joint profit of dyads in each group.

\section{Results}

\section{Descriptive statistics}

The means and standard deviations of the joint profit for each group as well as the dyad composition of each group are presented in Table 2 below. Figure 1 presents the means and standard deviations visually. Over a four-month period, a total of 120 participants (60 dyads) were invited back for the experiment. As shown in Table 2, we have a very low sample size in the group with low CQ dyads $(N=3)$. The second smallest group was the group with high CQ domestic - low CQ international student dyads $(N=6)$. The small sample size for these two groups was due to 
difficulty in recruiting low CQ international participants as our international participants sample have relatively high CQ. In fact, results from Stage 1 indicated that the international participants had significantly higher overall CQ compared to the domestic participants $(t(799)=4.99, p<.001$; $\left.M_{\text {international }}=4.87, S D_{\text {international }}=.72 ; M_{\text {domestic }}=4.61, S D_{\text {domestic }}=.74\right)$, making it harder to recruit low CQ compared to high CQ international participants.

Furthermore, of the 60 dyads that participated in the experiment (Stage 2), only 40 were included in the analyses reported below. The 40 dyads included for all subsequent analyses were composed of strictly Asian/Pacific and New Zealand European pairs. There were two main reasons for excluding 20 dyads from our analysis. Firstly, dyads that, upon further inspection, did not fit the intended categories were discarded. Dyads that did not fit the intended categories include dyads comprising New Zealand Europeans of mixed cultural heritage and Americans and western Europeans. This occurred due to participants not indicating their cultural heritage/affiliation appropriately during the pre-screening study (Stage 1). Another reason why dyads were discarded was due to participant no-shows. Given that we required two participants for each negotiation, in cases where only one participant attended their experimental session, we were forced to make impromptu arrangements for another participant to make up the dyad. However, this sometimes resulted in dyads that did not fit the intended categories and we had to later exclude from analysis. 
Group

Low CQ domestic - Low CQ international student

dyad

Low CQ domestic - High CQ international student

12

dyad

High CQ domestic - Low CQ international student

dyad

High CQ domestic - High CQ international student

dyad
545.38

81.38

$\mathrm{N}$

3

590.00

70.71

$S D$

6

528.33

38.17

19

609.47

60.07

\section{Total}

40

575.50

72.36

Table 2. Means and standard deviations of group joint profit

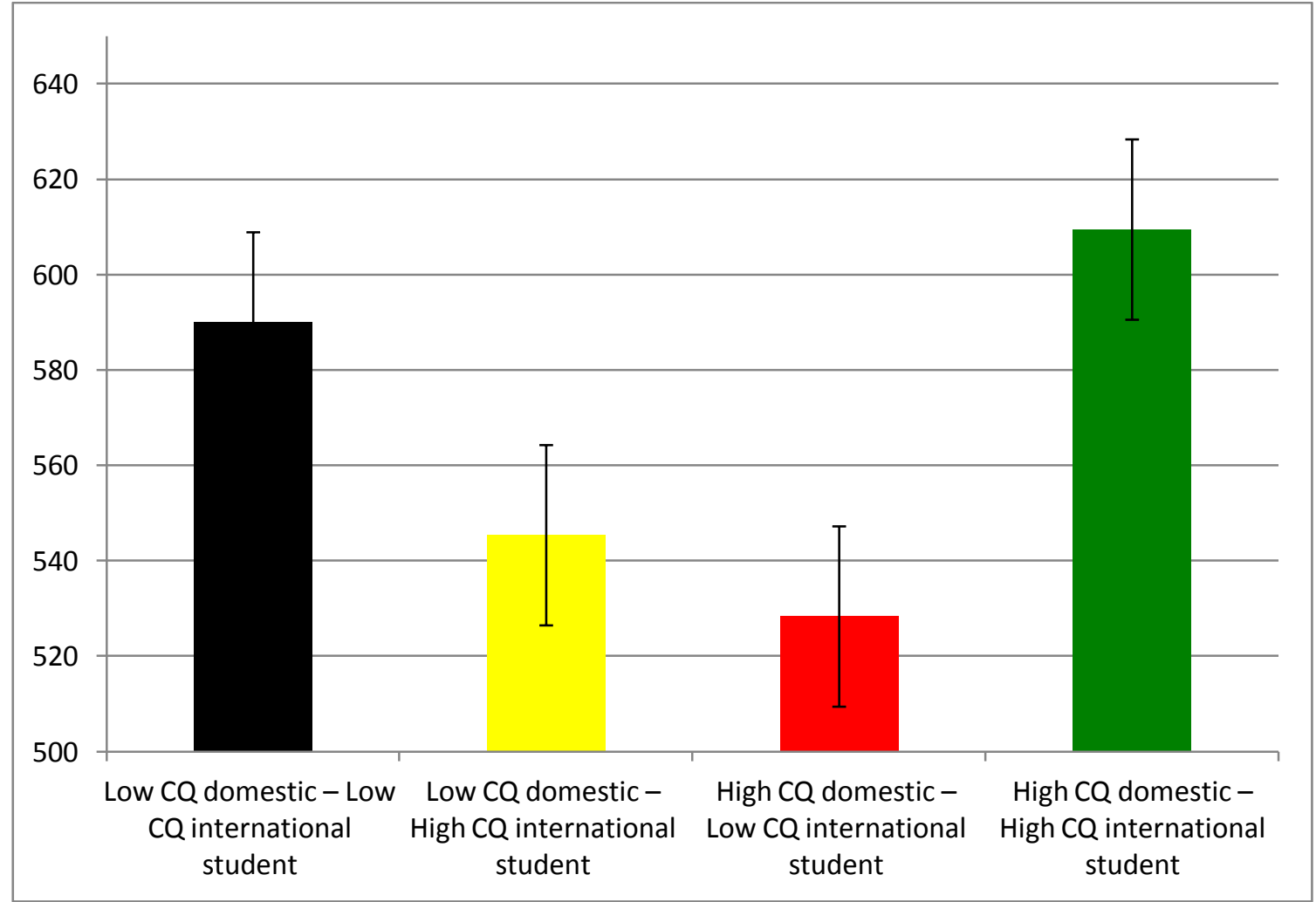

Figure 1. Bar graph presenting mean joint profits.

\section{Manipulation check}

A manipulation check was conducted to confirm that high CQ individuals from the pre- 
screening test (Stage 1) still had significantly higher CQ scores compared to the low CQ individuals during the experiment (Stage 2). An independent samples t-test confirmed that low and high CQ individuals still have significantly different CQ scores, $t(77)=-7.12, p<.001$. The results indicate that high CQ individuals $(M=5.32, S D=.61)$ had significantly higher scores on the CQ scale compared to individuals with low CQ $(M=4.36, S D=.57)$ during the experiment (Stage 2$)$.

Tests of hypotheses

Following Imai and Gelfand's (2010) procedure, all individual difference scores were aggregated to the dyad-level by averaging each dyad's scores prior to hypothesis testing.

Hypothesis 1 predicted that high CQ dyads will achieve significantly higher joint profits compared to dyads with at least one low CQ negotiator. An analysis of variance (ANOVA) found a significant main effect of group, $F(3,36)=3.64, p=.02, \eta_{\mathrm{p}}{ }^{2}=.23$, with dyads in the high CQ group achieving the highest joint profits $(M=609.47, S D=60.07$ out of the four groups. Simple contrasts conducted found that dyads in the two mixed-CQ groups: low CQ domestic - high CQ international students (contrast estimate $=-64.09, t(30)=-2.57, p=.02)$ and high CQ domestic - low CQ international students (contrast estimate $=-81.14, t(23)=-3.09, p=.005)$ achieved significantly lower joint profits compared to dyads in the high CQ group. However, dyads in the low CQ group did not achieve significantly lower joint profit compared to dyads in the high CQ group (contrast estimate $=-19.47, t(19)=-.43, p=.67)$. This non-significant result may be due to the small sample size in the low CQ dyads group.

Because of the small sample size in the low CQ dyads group, a separate ANOVA was conducted excluding low CQ dyads group to determine if the significant main effect of group still held. The main effect of group was still significant, $F(2,35)=5.43, p=.009, \eta_{\mathrm{p}}{ }^{2}=.24$, after removing the small low CQ dyads group. We therefore included all four groups in subsequent analyses.

Hypothesis 2 predicted that CQ predicts significantly higher joint profits above and beyond individual difference characteristics associated with negotiation effectiveness in 
intracultural contexts. Due to the small overall sample size $(N=40)$, seven separate analysis of covariance (ANCOVA) were conducted to test this hypothesis instead of conducting one ANCOVA controlling for all covariates at once. Results of the ANCOVA are provided in Table 3, which summarises the main effect of $\mathrm{CQ}$ while controlling for each of the individual difference characteristics.

\begin{tabular}{lcccc}
\hline Main effect of CQ controlling for: & $F$ & $d f$ & $p$-value & $\eta_{\mathrm{p}}{ }^{2}$ \\
\hline Emotional intelligence (EQ) & 3.20 & 3,35 & .04 & .22 \\
Cognitive ability (IQ) & 2.91 & 3,35 & .048 & .20 \\
Extraversion & 4.17 & 3,35 & .01 & .26 \\
Openness to Experience & 3.78 & 3,35 & .02 & .25 \\
Agreeableness & 3.57 & 3,35 & .02 & .23 \\
Conscientiousness & 3.53 & 3,35 & .03 & .23 \\
Emotional Stability & 3.65 & 3,35 & .02 & .24 \\
Table 3. Ancova: Summary of group main effect while controlling for individual difference characteristics.
\end{tabular}

Consistent with Hypothesis 2, results from the ANCOVAs indicated that CQ remains a significant predictor of joint profit even after controlling for emotional intelligence, cognitive ability, and the Big Five personality traits (extraversion, openness to experience, agreeableness, conscientiousness, and emotional stability). None of the control variables significantly predicted joint profit: IQ $(F(1,35)=.47, p=.50), \mathrm{EQ}(F(1,35)=1.12, p=.30)$, emotional stability $(F(1,35)$ $=.25, p=.62)$, extraversion $(F(1,35)=1.53, p=.22)$, openness to experience $(F(1,35)=.88, p$ $=.35)$, agreeableness $(F(1,35)=.72, p=.40)$, and conscientiousness $(F(1,35)=.01, p=.30)$.

In order to examine which of the four CQ factor is driving the effects of higher joint profit, additional regression analyses were conducted. Stepwise regression revealed that motivational CQ was the only factor which significantly predicted joint profit, $\beta=.32, t(38)=2.05$, $p=.048$. However, when joint profit was regressed on all four CQ factors at once (enter method of 
regression), none of the CQ factors significantly predicted joint profit, $F(4,35)=1.17, p=.34$. The unique variance associated with motivational $\mathrm{CQ}$, which had the overall highest correlation with joint profit, was not significant when taking into account its shared variance with the other three CQ factors (i.e., metacognitive CQ, cognitive $C Q$, and behavioural CQ).

Finally, no main effects of group were found for levels of frustration $(F(1,36)=.1 .12, p$ $=.36)$ or perceived cooperation $(F(1,36)=.38, p=.77)$. The experimental groups did not differ in terms of perceived cooperation and level of frustration. Furthermore, no main effects of group were found for social attraction $(F(3,36)=.57, p=.64)$. Once again, the experimental groups did not differ in reported levels of social attraction towards their negotiation partner.

\section{Sequences of integrative information negotiation}

Out of the overall sample $(N=40)$, only three dyads engaged in sequences of integrative information behaviours, and no dyad engaged in sequences of cooperative relationship management. Two of these dyads were composed of high CQ negotiators, whereas the remaining dyad was in the low CQ domestic - high CQ international group. Nevertheless, it should be noted that Imai and Gelfand (2010) also reported very low frequencies of integrative information and cooperative relationship management sequences in their study $\left(M_{\text {Reciprocal }}=.02, S D_{\text {Reciprocal }}=.03\right.$; $\left.M_{\text {Complementary }}=.02, S D_{\text {Complementary }}=.02 ; M_{\text {Cooperative }}=.04, S D_{\text {Cooperative }}=.04\right)$. Following Imai and Gelfand's (2010) example, we converted the raw frequencies for each type of strategic sequence to control for the total number of speaking turns across dyads (i.e., we controlled for the length of the negotiation). Each raw frequency was divided by the dyad's total number of speaking turns minus one. Table 4 summarises the raw and relative frequencies of integrative negotiation sequences engaged by the three dyads as well as each dyad's joint profits. 


\begin{tabular}{|c|c|c|c|c|c|}
\hline Dyad & $\begin{array}{l}\text { Aggregated } \\
\text { joint profit }\end{array}$ & $\begin{array}{c}\text { Reciprocal } \\
\text { sequences } \\
\text { (Raw) }\end{array}$ & $\begin{array}{l}\text { Reciprocal } \\
\text { sequences } \\
\text { (Relative) }\end{array}$ & $\begin{array}{l}\text { Complementary } \\
\text { sequences } \\
\text { (Raw) }\end{array}$ & $\begin{array}{l}\text { Complementary } \\
\text { sequences } \\
\text { (Relative) }\end{array}$ \\
\hline 26 & 660 & 0 & 0 & 2 & .04 \\
\hline 28 & 540 & 3 & .05 & 0 & 0 \\
\hline 57 & 505 & 2 & .05 & 0 & 0 \\
\hline
\end{tabular}

As shown in Table 4, our sample engaged in fewer negotiation sequences of integrative information compared to Imai and Gelfand (2010). The mean for complementary sequences of integrative information behaviours was $.001(S D=.007)$ and the mean for reciprocal sequences of integrative information behaviours was $.003(S D=.011)$. Furthermore, the negotiation outcomes (i.e., joint profits) of the three dyads that engaged in integrative information sequences did not appear to be different from dyads that did not engage in integrative information sequences. Although dyad 26 achieved joint profits more than one standard deviation from the mean overall joint profit $(M=575.50, S D=72.36)$, the remaining two dyads $(28$ and 57$)$ achieved joint profits within one standard deviation of the overall mean joint profit. Based on the low base rate of integrative information sequences and similar negotiation outcomes between dyads that engaged in sequences of integrative negotiation sequences and dyads that did not, we decided that further analyses would not reveal meaningful/interpretable results.

\section{Discussion}

To the best of my knowledge, this research project was the first study to experimentally manipulate dyad compositions to examine the effect of cultural intelligence (CQ) on intercultural negotiation. Consistent with Imai and Gelfand's (2010) correlational findings, results from our experiment support the prediction that high CQ dyads will achieve significantly higher joint profits 
compared to dyads with at least one low CQ negotiator. Our second hypothesis, that CQ predicts significantly higher joint profits beyond individual characteristics associated with intracultural negotiation effectiveness was also supported. Our results indicate that CQ was able to predict intercultural negotiation effectiveness over and above individual characteristics including cognitive ability (e.g., Fulmer \& Barry, 2004; Barry \& Friedman, 1998; Kurtzberg, 1998), emotional intelligence (e.g., Elfenbein et al., 2007, Der Foo et al., 2004, Fulmer \& Barry, 2004), and the Big 5 personality traits: extraversion (e.g., Cullen et al., 2013; Nauta \& Sanders, 2000; Barry \& Friedman, 1998,), agreeableness (e.g., Nauta \& Sanders, 2000; Barry \& Friedman, 1998), conscientiousness (e.g., Cullen et al., 2013), and openness to experience (e.g., Ma \& Jaeger, 2005, 2010). Additionally, previous research on negotiator characteristics that may predict negotiation outcomes was predominantly conducted in monocultural or comparative settings (e.g., Ma \& Jaeger, 2010).

Notably, none of these individual characteristics that previously predicted negotiation outcomes in mono-cultural settings was significantly related to intercultural negotiation effectiveness. Our findings suggested that individual characteristics that were found to predict intracultural negotiation effectiveness may not be an advantage in intercultural negotiation settings. The results supported findings by Imai and Gelfand (2010) who found that none of the individual characteristics predicted sequences of integrative information and cooperative relationship management behaviours which then predicted higher joint profit. These findings further highlight the importance of not assuming that individuals' behaviours (or individual characteristics advantageous for negotiation effectiveness) remain the same across intracultural and intercultural negotiation contexts (Adler \& Graham, 1989).

Examining which of the CQ factors may be the strongest predictor of intercultural negotiation effectiveness, we found that none of the CQ factors individually predicted optimal intercultural negotiation outcomes. The findings suggested that it is overall CQ that is driving effective intercultural negotiation (i.e., higher joint profit). This finding lends support to the conceptualisation of CQ as a system which was put forth by Thomas and colleagues (Thomas et al., 
2008). They defined CQ as a system of interacting knowledge and skills that allows people to adapt to environments characterised by cultural diversity and cross-cultural interactions (Thomas et al., 2008). What is distinct in their definition of CQ is that the researchers view CQ as a construct that emerges as a result of the interaction of its factors (Thomas et al., 2008). However, not much is currently known regarding the processes by which each CQ factor interact in order to produce culturally intelligent behaviour (Gelfand et al, 2008). More research needs to be done in order to examine how the factors work together to allow individuals to adapt to culturally diverse environments.

Nevertheless, we were able to rule out alternative explanations of how CQ affects intercultural negotiation effectiveness. Our findings indicate that overall CQ does not lead to increased cooperation or social attraction. Furthermore, results suggest that overall CQ does not lead to decreased frustration towards one's partner during the negotiation. Taken together, the results suggest that there are other underlying processes mediating the relationship between CQ and intercultural negotiation effectiveness.

Our final two hypotheses predicted that: a) high CQ dyads will engage in significantly more integrative information behaviour strategies (both reciprocal and complementary) compared to dyads with at least one low CQ negotiator (Hypothesis 3); and b) high CQ dyads will engage in significantly more cooperative relationship management behaviours compared to dyads with at least one low CQ negotiator (Hypothesis 4). Similar to Imai and Gelfand's study (2010), our sample showed very few sequences of integrative information behaviours (reciprocal and complementary). Only three dyads in our sample showed reciprocal and complementary sequences of integrative information behaviours and no dyads showed sequences of cooperative relationship management behaviours. Despite the low frequencies of integrative information behaviour sequences (complementary and reciprocal) and sequences of cooperative relationship management behaviours, Imai and Gelfand (2010) further conducted a logit-transformation to stretch the tails of the distribution of the relative frequencies of each strategic negotiation sequence (complementary, 
reciprocal, and cooperative). However, given the low base rate of integrative information sequences in our sample and similar negotiation outcomes between dyads that engaged in sequences of integrative negotiation sequences and dyads that did not, we were unable to formally test these hypotheses. The low base rates of integrative information sequences overall did not confirm Hypotheses 3 and 4.

Furthermore, our results indicated that the two mixed-CQ groups (low CQ domestic high CQ international and high CQ domestic - low CQ international student dyads) achieved significantly lower joint profits than the group with high CQ dyads. This suggested that both negotiators need to have high levels of CQ in order to realise optimal intercultural negotiation outcomes. The dyad composition effect thus highlights a potential limitation of CQ as a predictor of intercultural negotiation effectiveness as CQ requires mutuality (i.e., both negotiators need to be equally high in CQ) in order to realise optimal negotiation effectiveness. According to the dyad composition effect, the lower CQ negotiator constrains the level of sequencing of integrative information and cooperative relationship management behaviours which then leads to higher joint profits (Imai \& Gelfand, 2010). Although we were unable to formally test Hypotheses 3 and 4 due to low base rates of integrative information behaviours, the finding that the two mixed-CQ groups performed worse than group with high CQ dyads suggests that the dyad composition effect still holds in our sample.

Our overall results support Imai and Gelfand's (2010) conclusion that cultural intelligence is a significant predictor of intercultural negotiation effectiveness beyond other individual characteristics found to predict negotiation effectiveness in intracultural negotiation settings. Consequently, our results demonstrated that cultural intelligence may be, as suggested by Imai and Gelfand (2010), the individual characteristic that negotiators can be selected and/or trained upon to specifically maximise intercultural negotiation effectiveness. Our findings further suggest that although sequences of integrative information behaviours did not function as predictors of higher joint profit in our sample, neither did they have any adverse effects on intercultural 
negotiation outcomes. Further research is therefore needed to examine the mechanisms through which CQ leads to optimal intercultural negotiation outcomes.

\section{Theoretical implications}

Our findings support the emerging view in the culture and negotiation literature that negotiators face different challenges and behave differently in intercultural compared to intracultural negotiation situations (e.g., Adler \& Graham, 1989; Brett \& Okumura, 1998; Adair et al., 2001; Adair, 2003). Results indicate that CQ was the sole predictor of intercultural negotiation effectiveness. Individual characteristics advantageous to intracultural negotiation effectiveness did not predict intercultural negotiation effectiveness. However, our results do not explain how CQ translates into more effective behaviours that lead to optimal outcomes during intercultural negotiation. Given that CQ predicted neither higher sequences of integrative information and cooperative relationship management behaviours nor increased understanding of one's partner's priorities, what exactly drove higher joint profits in the high CQ dyads? Further research is needed to examine the mechanisms through which overall CQ predicts intercultural negotiation effectiveness and the processes by which different factors of CQ interact in order to produce culturally intelligent behaviour.

One possible explanation for the CQ - intercultural negotiation effectiveness relationship is that dyads with higher CQ engage in other non-verbal and/or paraverbal communication during the negotiation which improved negotiation effectiveness (i.e. higher joint profits). Paraverbal communication refers to pauses, loudness, interruptions, and use of nonlanguage sounds during an interaction (Adair \& Brett, 2004). It is important to remember that communication occurs through both verbal and non-verbal means. Poon (1998) claimed that any analysis of communication is incomplete without examining the non-verbal aspects of communication. It is possible that CQ predicts optimal intercultural negotiation outcomes through both the verbal sequences of integrative information and cooperative management behaviours and the appropriate use of nonverbal and paraverbal communication. Considering that culturally 
intelligent behaviour has been conceptualised as a crucial aspect of CQ (e.g., Earley \& Ang, 2003; Earley et al., 2006; Ang et al., 2007; Ang \& Van Dyne, 2008; Thomas et al., 2008), it is likely that nonverbal behaviour would play as much a role in predicting effective intercultural negotiation as verbal behaviour. For example, a category of nonverbal behaviours called regulators, which are gestures, movements of the head, facial expressions and posture that indicate one's interest and a desire for the speaker to speak faster or slower, have been described as one of the most culturally determined ways of communicating non-verbally (Poon, 1998). Thus, when investigating what drives effective intercultural negotiation, it is important to differentiate between intelligence and culturally intelligent behaviour. It has been noted that although intelligent behaviours can be construed differently across cultures (e.g., Johnson, Lenartowicz, \& Apud, 2006), the same mental processes may produce different behaviours depending on the cultural context (Thomas et al., 2008). It may be that individuals with high CQ were more equipped to draw upon these mental processes to determine culturally appropriate behaviours while negotiating in an intercultural setting. Further research is needed to examine the specific verbal and nonverbal behaviours that are driving integrative strategies for more effective intercultural negotiations.

Another potential factor that can help explain how CQ affects intercultural negotiation effectiveness could be the interaction between the negotiation context and negotiator characteristics. There has been an emerging focus in the negotiation literature examining the bi-directional relationship between negotiator characteristics (e.g., personality, cognitions, interaction styles) and contextual effects (e.g., structural features of a negotiation setting) and how that influences negotiation outcomes (see review by Li, Tost, \& Wade-Benzoni, 2006). The dyad composition effect indicates that intercultural negotiators need to have similarly high levels of overall CQ in order to achieve higher joint profits; highlighting negotiator characteristics that are advantageous for effective intercultural negotiation. However, the optimal structural features in an intercultural negotiation setting that will strengthen the effects of overall CQ on negotiation outcomes are still unknown. Moreover, a meta-analytic review on mono-cultural negotiation settings indicated that 
negotiators with prosocial orientations tend to achieve higher joint profits, especially in conditions of high resistance to yielding (De Dreu, Weingart, \& Kwon, 2000). Other research linking prosocial values and orientations and cooperative behaviours have also suggested that, when negotiators are more prosocial (rather than pro-self), they tend to engage in more cooperative problem-solving behaviours that lead to higher joint profits (e.g., Olekalns \& Smith, 2003; De Dreu, Beersma, Stroebe, \& Euwema, 2006). The literature on prosocial orientation and cooperative behaviours could help to explain previous findings suggesting that intercultural negotiators achieve lower joint gains compared to intracultural negotiators because intercultural negotiators are not motivated to achieve integrative solutions (e.g., Brett \& Okumura, 1998; Adair et al., 2001).

The moderating effect of prosocial or pro-self conditions on the relationship between CQ and intercultural negotiation effectiveness can potentially be explained using the dual concern model (Pruitt \& Kim, 2004). The dual concern model posits two types of concerns, self-concern (concern about one's own outcomes) and other concern (concern about the partner's outcomes), which ranges from weak to strong (Pruitt \& Kim, 2004). Pruitt and Kim (2004) argue that the model can serve as a theory about the impact of conditions on strategic choice. For example, the dual concern model predicts that problem solving is encouraged when one has high self- and other concern such as when a negotiator is instructed to cooperate rather than compete in order to generate integrative solutions (Pruitt \& Lewis, 1975; Pruitt \& Kim, 2004). Future studies could manipulate conditions to promote prosocial or pro-self orientations in negotiators in order to test whether prosocial orientation moderates the effect of overall CQ on intercultural negotiation effectiveness.

\section{Practical Applications}

One of the main practical implications of our findings was that intercultural negotiators need to have similarly high levels of CQ in order to achieve optimal intercultural negotiation outcomes. Given the dyad composition effect, intercultural training to improve CQ needs to occur at both ends of the communication 'stream'. More importantly, considering the finding that 
international students have higher CQ compared to domestic students, it appears that domestic students are the constraining factor in achieving optimal negotiation outcomes. Given these findings, it is likely that incoming migrants will also tend to have higher CQ compared to host nationals. Therefore, it is not enough for countries to emphasise solely on providing destination information to incoming migrants (e.g., the customs, norms, and laws of the destination countries). It is just as important for the destination countries to learn about the people entering its borders and to train host nationals (i.e., civil servants who frequently interact with migrants) and equip them with the knowledge and skills to communicate effectively across cultures.

Our findings also indicated that cultural intelligence may be the individual characteristic that negotiators can be trained and/or selected to specifically maximise intercultural negotiation effectiveness. Given that CQ has been conceptualised both as a capability (Earley \& Ang, 2003) and a system of interacting knowledge and skills (Thomas et al., 2008) that allows an individual to perform and manage effectively in situations characterised by cultural diversity, it should therefore be possible to provide training aimed at improving individuals' CQ. Indeed, given the increase in global mobility due to globalisation and the ease of cross-border communication, it is important that managers, students, and diplomats (among others) are trained to improve their cultural intelligence in order to facilitate cross-cultural adjustment and performance.

As CQ is still a relatively new construct, there are currently few studies which have specifically examined the effectiveness of intercultural training programmes in improving CQ (Rehg, Gundlach, \& Grigorian, 2012). To the best of my knowledge, the literature on the effectiveness of intercultural training in improving CQ has yielded mixed findings (e.g., Rehg et al., 2012, Fischer, 2011). For example, Rehg and colleagues (Rehg et al., 2012) found that lecture-based intercultural training could significantly improve cognitive and behavioural CQ in American government contracting trainees. On the other hand, research by Fischer (2011) found that participants reported significant declines in cognitive and metacognitive CQ after a six-week intercultural training intervention incorporating a combination of lectures, simulation and 
behavioural training to raise awareness about cultural differences in the context of a university course. Participant feedback indicated that although the experiential training sessions were stimulating and challenging, the training sessions also resulted in a growing awareness of cultural differences and participants' awareness of their shortcomings in their abilities and skills to interact effectively across different cultural contexts (Fischer, 2011).

However, given that the two intercultural training interventions by Fischer (2011) and Rehg and colleagues (Rehg et al., 2012) were conducted for two different purposes, the mixed findings may actually paint a clearer picture of the effectiveness of intercultural training programmes in improving CQ. The lecture-based intercultural training by Rehg and colleagues (Rehg et al., 2012) was designed as part of a training course for US government contracting trainees being trained for overseas assignments whereas the intercultural training intervention conducted by Fischer (2011) was aimed to raise awareness of cultural differences in the context of a university course. As such, the lecture content was designed to focus on helping trainees develop a basic understanding of culture, with an emphasis on the destination country (Rehg et al., 2012). The culture-specific training content was thus able to improve levels of cognitive and behavioural CQs. On the other hand, the intercultural training intervention by Fischer (2011) was more focused on culture theory and activities designed to raise awareness on cultural differences. As such, the decline in levels of cognitive and meta-cognitive CQ reported by students could be interpreted as an increasing consciousness of their limits to interacting effectively in culturally diverse situations. Fischer (2011) calls the intervention as a 'reality check' of one's abilities and skills of negotiation different cultural contexts.

The available research examining the effectiveness of intercultural training programmes in improving CQ to date indicates that a combination of methods (i.e., mixture of lecture-based and experiential strategies) may be more effective in improving one's CQ. The experiential component of an intercultural training intervention could function to raise participants' awareness of cultural differences and the importance of cultural competence. Additionally, given that participants reported 
experiential sessions to be more stimulating and challenging, the experiential component could also function to further motivate (i.e., increase one's motivational CQ) to learn more about other cultures. Alternatively, the lectures could serve to increase one's knowledge of culture theory and specific cultural values and norms. Cultural assimilators could also be employed to supplement these lectures. Culture-specific assimilators are programmed learning experiences designed as a means of exposing members of a culture to some of the basic concepts, values, attitudes, and customs of another culture (Fiedler, Mitchell, \& Triandis, 1971). Research by Bhawuk (1989) suggests that cultural assimilators are an important part of cross-cultural training programmes, as they increase intercultural sensitivity, accuracy in making attributions in intercultural interactions, and training programme satisfaction.

Limitations, strengths, and future research

One of the main limitations of the current study was the uneven group sizes across the four groups. The two smallest groups were the groups consisting low CQ dyads and high CQ domestic - low CQ international student dyads. As was observed during the pre-screening study (Stage 1), the overall international student sample had significantly higher CQ compared to the domestic student sample. As a result, it was extremely difficult to identify and recruit low CQ international participants for the experiment at Stage 2. Further analyses have shown that excluding the smallest group (low CQ dyads) did not change our results. CQ was still a significant predictor of intercultural negotiation effectiveness even after excluding the smallest group. Future research should ensure a more even number of dyads in each group. Furthermore, as pointed out by Imai and Gelfand (2010), the majority of research in intercultural negotiation has focused on Americans and East Asians. Our experiment went beyond this to examine New Zealand European and Asian and Pacific cultures. However, it would be beneficial to further determine whether CQ as a predictor of intercultural negotiation effectiveness extends to intercultural negotiations between members of other cultures as well (Imai \& Gelfand, 2010). Given that CQ is not culture specific, but a situationspecific capability (i.e., culturally diverse situations) (Ang \& Van Dyne, 2008), its effects on 
intercultural negotiation effectiveness should also extend to members of other cultures.

To the best of our knowledge, the present research was the first to experimentally examine CQ as a predictor of intercultural negotiation effectiveness by manipulating the CQ level of dyads. Apart from allowing greater internal control, the experimental design also allows us to draw causal links between CQ and intercultural negotiation outcomes. Our results thus allow us to conclude that CQ leads to greater joint profits. Since higher CQ did not predict greater sequencing of integrative information and cooperative relationship management behaviours, we have yet to determine the process by which CQ leads to optimal intercultural negotiation outcomes. As previously discussed, CQ may lead to intercultural negotiation effectiveness through the use of appropriate nonverbal and paraverbal communication. Future research should examine the roles of verbal, non-verbal, and paraverbal communication in intercultural negotiation outcomes.

Furthermore, considering the mixed findings in the intercultural training literature thus far (e.g., Fischer, 2011; Rehg et al., 2012), future research could also seek to determine whether intercultural training really does improve CQ by collecting both observer and self-report CQ, before and after the intercultural training programme. If both self and observer reports of CQ converge at both time points, it would further strengthen the use of self-report measures in the research. However, divergence of self and observer-reported CQ post-training could lead to some interesting conclusions. For example, if self-reported CQ declines (as found in Fischer, 2011) but observerreported CQ increases post-training, it could mean that the intercultural intervention had served as a 'reality check' of one's intercultural abilities and skills but the observer-reported CQ would indicate that the individual has indeed improved over the course of the intervention.

Finally, although participants were instructed to work towards integrative negotiation outcomes (i.e., they were instructed to maximise profits for themselves and their negotiation partner), actual prosocial orientations were not measured. Additionally, previous research have found that prosocial negotiators tend to engage in more cooperative problem-solving behaviours that lead to higher joint profits (e.g., Olekalns \& Smith, 2003; De Dreu et al., 2000, 2006). As all 
participants in the study received grocery vouchers as a token of appreciation, future experiments examine the role of prosocial and pro-self orientations in moderating the link between CQ and intercultural negotiation effectiveness by making rewards (e.g., value of voucher) contingent on either joint or personal profit in order to manipulate cooperative and competitive motivations.

\section{Conclusion}

We were partially successful in replicating Imai and Gelfand's (2010) study examining CQ as a predictor of intercultural negotiation effectiveness. To the best of our knowledge, this research is the first to experimentally examine CQ as a predictor of intercultural negotiation effectiveness. Our results indicate that CQ was able to predict intercultural negotiation effectiveness even though other individual characteristics identified in the negotiation literature to predict effective intracultural negotiation did not. This lends support to the view that negotiators need a different set of skills to function effectively in intercultural and intracultural negotiation situations in order to achieve optimal integrative outcomes. Furthermore, our results indicated overall CQ, rather than individual CQ factors, is driving effective intercultural negotiation effectiveness. Contrary to Imai and Gelfand's (2010) findings, we found that higher CQ did not lead to more sequencing of integrative information and cooperative relationship management behaviours which then led to higher joint profits. Future research is needed in order to examine how individual CQ factors interact to produce culturally intelligent behaviour and the processes by which CQ leads to optimal intercultural negotiation outcomes. 


\section{References}

Adair, W. L., Okumura, T., \& Brett, J. M. (2001). Negotiation behaviour when cultures collide: The United States and Japan. Journal of Applied Psychology, 86(3), 371 - 385. doi: 10.1037//0021-9010.86.3.371.

Adair, W. L. (2003). Integrative sequences and negotiation outcome in same- and mixed-culture negotiations. International Journal of Conflict Management. 14(3/4), 273 - 296.

Adair, W. L. \& Brett, J. M. (2004). Culture and negotiation processes. In M. J. Gelfand \& J. M. Brett (Eds.), The Handbook of Negotiation and Culture (pp.143 - 157). Palo Alto, CA: Stanford University Press.

Adair, W. L. \& Brett, J. M. (2005). The negotiation dance: Time, culture, and behavioral sequences in negotiation. Organization Science, 16(1), 33 - 51. doi: 10.1287/orse.1040.0102.

Adler, N. J. \& Graham, J. L. (1989). Cross-cultural interaction: The international comparison fallacy? Journal of International Business Studies, 20(3), 515 - 537.

Ang, S., Van Dyne, L., \& Koh, C (2006). Personality correlates of the four-factor model of cultural intelligence. Group \& Organisation Management, 31, 100 - 123. doi: $10.1177 / 1059601105275267$

Ang, S., Van Dyne, L., Koh, C., NG, K. Y., Templer, K., Tay, C., \& Chandrasekar, N. A. (2007). Cultural intelligence: Its measurement and effects on cultural judgment and decision making, cultural adaptation and task performance. Management and Organization Review, $3,35-371$.

Ang, S. \& Van Dyne, L. (2008). Conceptualisation of cultural intelligence: Definition, distinctiveness, and nomological network. In S. Ang \& L. Van Dyne (Eds.), Handbook of cultural intelligence: Theory, measurement, and applications (pp. 3 -15). Armonk, NY: M.E. Sharpe. 
Ang, S., Van Dyne, L., \& Tan, M. L. (2011). Cultural Intelligence. In R. J. Sternberg \& S. B. Kaufman (Eds.). Cambridge Handbook on Intelligence (pp. 582 - 602). New York: Cambridge Press.

Bandura, A. (2002). Social cognitive theory in cultural context. Applied Psychology: An international review, 51(2), $269-290$.

Barry, B. \& Friedman, R. A. (1998). Bargainer characteristics in distributive and integrative negotiation. Journal of Personality and Social Psychology, 74(2), 345 - 359. Retrieved from PsycINFO.

Bhawuk, D. P. S. (1998). The role of culture theory in cross-cultural training: A multimethod study of culture-specific, culture-general, and culture theory-based assimilators. Journal of Cross-Cultural Psychology, 29, 630 - 655. doi: 10.1177/0022022198295003.

Brett, J. M. \& Okumura, T. (1998). Inter- and intracultural negotiation: US and Japanese negotiators. Academy of Management Journal. 41(5). 495 - 510

Chen, A. S., Lin, Y., \& Sawangpattanakul, A. (2011). The relationship between cultural intelligence and performance with the mediating effect of culture shock: A case from Philippine labourers in Taiwan. International Journal of Intercultural Relations, 35, $246-258$.

Cullen, M. J., Muros, J. P., Rasch, R., \& Sackett, P. R. (2013). Individual differences in the effectiveness of error management training for developing negotiation skills. International Journal of Selection and Assessment, 21(1), 1 - 21. Retrieved from PsycINFO.

De Dreu, C. K. W., Weingart, L. R., \& Kwon, S. (2000). Influence of social motives on integrative negotiation: A meta-analytic review and test of two theories. Journal of Personality and Social Psychology, 78(5), 889 - 905. doi: 10.1037//0022-3514.78.5.889.

De Dreu, C. K. W., Beersma, B., Stroebe, K., \& Euwema, M. C. (2006). Motivated information processing, strategic choice, and the quality of negotiated agreement. Journal of 
Personality and Social Psychology, 90(6), 927 - 943. doi: 10.1037/00223514.90.6.927.

Deci, E. L. (1975). Intrinsic Motivation. New York: Plenum Press.

Der Foo, M., Elfenbein, H. A., Tan, H. H., \& Aik, V. C. (2004). Emotional intelligence and negotiation: The tension between creating and claiming value. The International Journal of Conflict Management, 15(4), 411 - 429. Retrieved from PsycINFO.

Earley, C. \& Ang, S. (2003). Cultural Intelligence: Individual interactions across cultures. Stanford, California: Stanford University Press.

Earley, C., Ang, S., Tan, J. S. (2006). CQ: Developing cultural intelligence at work. California: Stanford University Pres.

Elfenbein, H. A., Der Foo, M., White, J., Tan, H. H., \& Aik, V. C. (2007). Reading your counterpart: The benefit of emotion recognition accuracy for effectiveness in negotiation. Journal of Nonverbal Behaviour, 31, 205 - 223. doi: 10.1007/s10919007-0033-7.

Fiedler, F. E., Mitchell, T., \& Triandis, H. C. (1971). The culture assimilator: An approach to cross-cultural training. Journal of Applied Psyschology, 55(2), 95 - 102. Retrieved from PsycINFO.

Fischer, R. (2011). Cross-cultural training effects on cultural essentialism beliefs and cultural intelligence. International Journal of Intercultural Relations. doi 10.1016/j.ijintrel.2001.08.005

Fischer, R. \& Fontaine, J. R. J. (2011). Methods for investigating structural equivalence. In D. Matsumoto \& F. J. R. Van de Vijver (Eds.), Cross-Cultural Research Methods in Psychology. New York: Cambridge University Press.

Flaskerud, J. H. (2007). Cultural competence: What is it? Issues in Mental Health Nursing, 28, 121 - 123. doi: 10.1080/01612840600998154.

Fulmer, I. S. \& Barry, B. (2004). The smart negotiator: Cognitive ability and emotional 
intelligence in negotiation. The International Journal of Conflict Management, 15(3), 245 - 272. Retrieved from PsycINFO.

Gelfand, M. J., Imai, L., \& Fehr, R. (2008). Thinking intelligently about cultural intelligence: The road ahead. In S. Ang \& L. Van Dyne (Eds.), Handbook of cultural intelligence: Theory, measurement, and applications (pp. 375 - 387). Armonk, NY: M.E. Sharpe.

Goldberg, L. R., Johnson, J. A., Eber, H. W., Hogan, R., Ashton, M., Cloninger, C. R., Gough, H. G. (2006). The international personality item pool and the future of public-domain personality measures. Journal of Research in Personality, 40, 84 - 96. doi: 10.1016/j.jrp.2005.08.007.

Harris, P. R. \& Moran, R. T. (1996). Managing Cultural Differences $\left(4^{\text {th }}\right.$ ed.). Houston: Gulf Publishing

Huff, K. C., Song, P., \& Gresch, E. B. (in press). Cultural intelligence, personality, and crosscultural adjustment: A study of expatriates in Japan. International Journal of Intercultural Relations. doi: 10.1016/j.ijintrel.2013.08.005.

Imai, L. \& Gelfand, M. J. (2010). The culturally intelligent negotiator: The impact of cultural intelligence (CQ) on negotiation sequences and outcomes. Organisational Behaviour and Human Decision Processes, 112, 83 - 98. doi: 10.1016/j.obhdp.2010.02.001

Johnson, J. P., Lenartowicz, T., \& Apud, S. (2006). Cross-cultural competence in international business: Toward a definition and a model. Journal of International Business Studies, 37, 525 - 543. Retrieved from PsycINFO.

Kurtzberg, T. R. (1998). Creativity thinking, cognitive aptitude, and integrative joint gain: A study of negotiator creativity. Creativity Research Journal, 11(4), 283 - 293. Retrieved from PsycINFO.

Li, M., Tost, L. P., \& Wade-Benzoni, K. (2006). The dynamic interaction of context and negotiator effects: A review and commentary on current and emerging areas in negotiation. International Journal of Conflict Management, 18(3/4), 222 - 259. doi: 


\section{$10.1108 / 10444060710825981$}

Lin, Y., Chen, A. S., \& Song, Y. (2012). Does your intelligence help to survive in a foreign jungle? The effects of cultural intelligence and emotional intelligence on cross-cultural adjustment. International Journal of Intercultural Relations, 36, 541 - 552. doi: 10.1016/j.ijintrel.2012.03.001.

Lun, V. M., Fischer, R., \& Ward, C. (2010). Exploring cultural differences in critical thinking: Is it about my thinking style or the language I speak? Learning and Individual Differences, 2010, 604 - 616. doi: 10.1016/j.lindif.2010.07.001.

Ma, Z. \& Jaeger, A. (2005). Getting to yes in China: Exploring personality effects in Chinese negotiating styles. Group Decision and Negotiation, 14, 415 - 437. doi: 10.1007/s10726-005-1403-3.

Ma, Z. \& Jaeger, A. (2010). A comparative study of the influence of assertiveness on negotiation outcomes in Canada and China. Cross Cultural Management: An International Journal, 17(4), 333 - 346. doi: 10.1108/13527601011086568.

McCroskey, L. L., McCroskey, J. C., \& Richmond, V. P. (2006). Analysis and improvement of the measurement of interpersonal attraction and homophily. Communication Quarterly, 54(1), 1 - 31. doi: 10.1080/01463370500270322.

Muthén, L. K. \& Muthén, B. O. (1998 - 2011). Mplus User’s Guide. Sixth Edition. Los Angeles, CA: Muthén \& Muthén.

Nauta, A. \& Sanders K. (2000). Interdepartmental negotiation behaviour in manufacturing organizations. The International Journal of Conflict Management, 11(2), 135 - 161. Retrieved from PsycINFO.

Olekalns, M. \& Smith, P. L. (2003). Social motives in negotiation: The relationships between dyad composition, negotiation processes, and outcomes. International Journal of Conflict Management, 14(3/4), 233 - 254. Retrieved from PsycINFO.

Poon, J. T. F. (1998). Nonverbal communication and business success. Management Research 
News, 21(4/5), 1 - 10. Retrieved from PsycINFO

Pruitt, D. G. \& Lewis, S. A. (1975). Development of integrative solutions in bilateral negotiation. Journal of Personality and Social Psychology, 31(4), 621 - 633.

Pruitt, D. G. \& Kim, S. H. (2004). Social Conflict: Escalation, stalemate, and settlement ( $3^{\text {rd }}$ edition). Boston: McGraw Hill.

Rehg, M. T., Gundlach, M. J., \& Grigorian, R. A. (2012). Examining the influence of crosscultural training on cultural intelligence and specific self-efficacy. Cross-Cultural Management, 19(2), 215 - 232. doi: 10.1108/13527601211219892.

Rockstuhl, T., Seiler, S., Ang, S., Van Dyne, L., \& Annen, H. (2011). Beyond general intelligence (IQ) and emotional intelligence (EQ): The role of cultural intelligence (CQ) on crossborder leadership effectiveness in a globalised world. Journal of Social Issues, 67(4), $825-840$.

Schutte, N. S., Malouff, J. M., Hall, L. E., Haggerty, D. J., Cooper, J. T., Golden, C. J., et al. (1998). Development and validation of a measure of emotional intelligence. Personality and Individual Differences, 25(2), 167 - 177.

Shipley, W. C. (1940). A self-administering scale for measuring intellectual impairment and deterioration. The Journal of Psychology: Interdisciplinary and Applied, 9(2), 371 377. doi: $10.1080 / 00223980.1940 .9917704$

Sternberg, R. J. (1986). A framework for understanding conceptions of intelligence. In R. J. Sternberg \& D. K. Detterman (Eds.). What is Intelligence? Contemporary viewpoints on its nature and definitions (pp. 3 - 15). Norwood, NJ: Ablex.

Templar, K. J., Tay, C., Chandrasekar, N. A. (2006). Motivational cultural intelligence, realistic job preview, realistic living conditions preview, and cross-cultural adjustment. Group and Organization Management, 31(1), 154 - 173. doi: 10.1177/1059601105275293.

Thomas, D. C., Elron, E., Stahl, G., Ekelund, B. Z., Ravlin, E. C., Cerdin, J., Poelmans, S., Brislin, R., Pekerti, A., Aycan, Z., Maznevski, M., Au, K., \& Lazarova, M. B. (2008). Cultural 
intelligence: Domain and assessment. International Journal of Cross Cultural Management, 8(2), 123 - 143. doi: 10.1177/1470595808091787.

Triandis, H. C. (1989). The self and social behaviour in differing cultural contexts. Psychological Review, 96(1), 506 -520. Retrieved from PsycINFO.

Tse, C. \& Altarriba, J. (2012). The effects of first- and second-language proficiency on conflict resolution and goal maintenance in bilinguals: Evidence from reaction time distributional analyses in a Stroop task. Bilingualism: Language and Cognition, 15(3), 663 - 676. doi: 10.1017/S1366728912000077.

Van Dyne, L., Ang, S. \& Koh, C. (2008). Development and validation of the CQS: The cultural intelligence scale. In S. Ang \& Van Dyne, L (Eds.), Handbook of cultural intelligence: Theory measurement, and applications (pp. 16-38). Armonk, NY: M.E. Sharpe.

Ward, C. \& Fischer, R. (2008). Personality, cultural intelligence CQ, and cross-cultural adaptation: A test of the mediation hypothesis. In S. Ang \& L. Van Dyne (Eds.), Handbook of Cultural Intelligence: Theory, measurement and applications (pp., 159 - 173). New York: M.E. Sharpe. Inc.

Ward, C., Fischer, R., Lam, F. S. Z., \& Hall, L. (2009). The convergent, discriminant, and incremental validity of scores on a self-report measure of cultural intelligence. Educational and Psychological Measurement, 69(1), 85 - 105.

Ward, C., Wilson, J., \& Fischer, R. (2011). Assessing the predictive validity of cultural intelligence over time. Personality and Individual Differences, 51, 138 - 142. doi: 10.1016/j.paid.2011.03.032.

Weingart, L. R., Prietula, M. J., Hyder, E. B., \& Genovese, C. R. (1999). Knowledge and the sequential processes of negotiation: A Markov chain analysis of response-in-kind. Journal of Experimental Social Psychology, 35, 366 - 393.

Weingart, L. R. \& Olekalns, M. (2004). Communication processes in negotiation: Frequencies, sequences, and phases. In M. J. Gelfand \& J. M. Brett (Eds.), The Handbook of 
Negotiation and Culture (pp.143 - 157). Palo Alto, CA: Stanford University Press.

Weingart, L. R., Olekalns, M., \& Smith, P. L. (2004). Quantitative coding of negotiation behaviour. International Negotiation, 9(3), 441 - 455.

Wigfield, A. \& Eccles, J. S. (2000). Expectancy-value theory of achievement motivation.

Contemporary Educational Psychology, 25, 68 - 81. doi: 10.1006/ceps.1999.1015. 


\section{Appendix}

\section{Section A: Negotiation task and role information}

\section{Towers Market II Participant Information Sheet: Jardin Florist}

You are the owner of Jardin Florist. In the past, your business has been mostly special orders and you are interested in using the market to demonstrate your unique style and to expand your clientele. You plan to specialise in made-to-order bouquets that will be made on the premises while customers wait.

A successful real estate developer has proposed developing a multi-functional market that includes a speciality grocery store and a florist with shared interior décor but with separate areas with which to sell their respective products. The market will have an open plan, with a single main entrance. This arrangement is advantageous for Jardin Florist not only because you can gain access to a larger group of consumers but also by sharing maintenance and upkeep costs of the building.

The developer has approached Jardin Florist to join the market. The developer plans to lease one floor of a primarily residential building for the market. The market will be located in an area which is currently being settled by young urban professionals - the potential customer pool.

The developer has asked the interested retailers to meet in order to decide on some of the issues that have to be resolved before any leases can be finalised, and before preparations to open the market can proceed. You are seriously interested in opening a shared market and have agreed to meet with Parducci's Grocery to try to resolve these issues so that preparations to open the market can move ahead. The issues that still need to be resolved with Parducci's Grocery are: a) hours of operation, b) renovation costs, c) floor space, d) temperature, and e) grand opening date.

See below for your confidential payoff schedule which outlines the options you have for each unresolved issue and the points associated with settling on each option. Please note that some of the issues are more important (i.e. worth more points) to you than others. 
The number of points associated with each level of settlement is confidential. Please do not share the number of points for each level of settlement with Parducci's Grocery. Your main goal is to maximise the number of points for both yourself and Parducci's Grocery. You may begin the negotiation when you and the owner of Parducci's are ready. You have a maximum of 15 minutes to negotiate.

\section{Payoff Schedule for Jardin Florist}

\begin{tabular}{|c|c|c|c|c|}
\hline Hours of Operation & $\begin{array}{c}\text { Renovation } \\
\text { Costs (each) }\end{array}$ & Floor Space & Temperature & Grand Opening \\
& & & \\
\hline $10: 30 \mathrm{am}-10: 30 \mathrm{pm}$ & $\$ 30,000$ & $40 \%$ for flowers & $18^{\circ} \mathrm{C}$ & June 1 \\
$(240$ points $)$ & $(400$ points $)$ & $(120$ points $)$ & $(200$ points $)$ & (80 points $)$ \\
\hline $9: 30 \mathrm{am}-9: 30 \mathrm{pm}$ & $\$ 25,000$ & $30 \%$ for flowers & $19^{\circ} \mathrm{C}$ & July 1 \\
$(180$ points $)$ & $(300$ points $)$ & $(90$ points $)$ & $(150$ points $)$ & $(60$ points $)$ \\
\hline $8: 30 \mathrm{am}-8: 30 \mathrm{pm}$ & $\$ 20,000$ & $50 \%$ for flowers & $20^{\circ} \mathrm{C}$ & August 1 \\
$(120$ points $)$ & $(200$ points $)$ & $(60$ points $)$ & $(100$ points $)$ & (40 points $)$ \\
\hline $7: 30 \mathrm{am}-7: 30 \mathrm{pm}$ & $\$ 15,000$ & $60 \%$ for flowers & $21^{\circ} \mathrm{C}$ & September 1 \\
$(60$ points $)$ & $(100$ points $)$ & $(30$ points $)$ & $(50$ points $)$ & $(20$ points $)$ \\
\hline 6:30am - 6:30pm & $\$ 10,000$ & $70 \%$ for flowers & $22^{\circ} \mathrm{C}$ & October 1 \\
$(0$ points $)$ & $(0$ points $)$ & $(0$ points $)$ & $(0$ points $)$ & (0 points $)$ \\
\hline
\end{tabular}


Participant no.:

Negotiation outcome: Jardin Florist

\begin{tabular}{|l|l|l|}
\hline Issue & Option chosen & My points \\
\hline Hours of Operation & & \\
\hline Renovation Costs & & \\
\hline Floor Space & & \\
\hline Temperature & & \\
\hline Grand Opening Date & & \\
\hline & & Total points = \\
\hline
\end{tabular}




\section{Towers Market II Participant Information Sheet: Parducci’s Grocery}

You are the owner of Parducci's Grocery. Parducci's Grocery is a successful gourmet grocery carrying a wide range of high quality imported grocery items and organically grown local produce. Parducci's has built a reputation for carrying exclusive imported brands and for supporting up and coming local New Zealand artisans.

A successful real estate developer has proposed developing a multi-functional market that includes a speciality grocery store and a florist with shared interior décor but with separate areas with which to sell their respective products. The market will have an open plan, with a single main entrance. This arrangement is advantageous for Parducci's not only because you can gain access to a larger group of consumers but also by sharing maintenance and upkeep costs of the building.

The developer had approached Parducci's join the market. The developer plans to lease one floor of a primarily residential building for the market. The market will be located in an area which is currently being settled by young urban professionals - the potential customer pool.

The developer has asked the interested retailers to meet in order to decide on some of the issues that have to be resolved before any leases can be finalised, and before preparations to open the market can proceed. You are seriously interested in opening a shared market and have agreed to meet with Jardin Florist to try to resolve these issues so that preparations to open the market can move ahead. The issues that still need to be resolved with Jardin Florist are: a) hours of operation, b) renovation costs, c) floor space, d) temperature, and e) grand opening date.

See below for your confidential payoff schedule which outlines the options you have for each unresolved issue and the points associated with settling on each option. Please note that some of the issues are more important (i.e. worth more points) to you than others.

The number of points associated with each level of settlement is confidential. Please do not share the number of points for each level of settlement with Jardin Florist. Your main goal is to 
maximise the number of points for both yourself and Jardin Florist. You may begin the negotiation when you and the owner of Jardin Florist are ready. You have a maximum of 15 minutes to negotiate.

\section{Payoff Schedule for Parducci's Grocery}

\begin{tabular}{|c|c|c|c|c|}
\hline $\begin{array}{c}\text { Hours of } \\
\text { Operation }\end{array}$ & $\begin{array}{c}\text { Renovation } \\
\text { Costs (each) }\end{array}$ & Floor Space & Temperature & Grand Opening \\
D:30am - 6:30pm & $\$ 10,000$ & $60 \%$ for grocery & $22^{\circ} \mathrm{C}$ & October 1 \\
$(240$ points $)$ & $(80$ points $)$ & $(120$ points $)$ & $(200$ points $)$ & $(400$ points $)$ \\
\hline $7: 30 \mathrm{am}-7: 30 \mathrm{pm}$ & $\$ 15,000$ & $70 \%$ for grocery & $21^{\circ} \mathrm{C}$ & September 1 \\
$(180$ points $)$ & $(60$ points $)$ & $(90$ points $)$ & $(150$ points $)$ & $(300$ points $)$ \\
\hline $8: 30 \mathrm{am}-8: 30 \mathrm{pm}$ & $\$ 20,000$ & $50 \%$ for grocery & $20^{\circ} \mathrm{C}$ & August 1 \\
$(120$ points $)$ & $(40$ points $)$ & $(60$ points $)$ & $(100$ points $)$ & $(200$ points $)$ \\
\hline $9: 30 \mathrm{am}-9: 30 \mathrm{pm}$ & $\$ 25,000$ & $40 \%$ for grocery & $19^{\circ} \mathrm{C}$ & July 1 \\
$(60$ points $)$ & $(20$ points $)$ & $(30$ points $)$ & $(50$ points $)$ & $(100$ points $)$ \\
\hline $10: 30$ am - & $\$ 30,000$ & $30 \%$ for grocery & $18^{\circ} \mathrm{C}$ & June 1 \\
$10: 30 \mathrm{pm}$ & $(0$ points $)$ & $(0$ points $)$ & $(0$ points $)$ & $(0$ points $)$ \\
$(0$ points $)$ & & & \\
\hline
\end{tabular}


Participant no.:

Negotiation outcome Parducci's Grocery

\begin{tabular}{|l|l|l|}
\hline Issue & Option chosen & My points \\
\hline Hours of Operation & & \\
\hline Renovation Costs & & \\
\hline Floor Space & & \\
\hline Temperature & & \\
\hline Grand Opening Date & & \\
\hline & & Total points = \\
\hline
\end{tabular}


Section B: Coding manual (adapted from Towers Market II coding manual)

$\underline{\text { Offer }}$

OS Single-issue - secure agreement on one issue

OM Multi-issue - secure agreement on two or more issues

Provide information

IP Issue preference - within a single issue

IR Priorities - relative importance of issue(s)

IB Bottom line - within a single issue or for a package

$\underline{\text { Substantiation }}$

SB Defending arguments - argue position on issue

SF Factual statements - facts or task clarifications that are specific/true

\section{Questions}

QP Ask for preference - within a single issue

QR Ask for priorities - relative importance of issue(s)

QB Ask for bottom line - within a single issue or package

QS Ask about substantiation - question/clarification of argument presented

QM Ask miscellaneous task-related questions

\section{$\underline{\text { Summarising }}$}

IN Insight - summarising others' interests

MU Mutuality - noting mutual interests

ID Differences - noting differences in issue-related preferences and priorities

IS Similarities - noting similarities in issue-related preferences and priorities 
GD General differences - which are task-related

GS General similarities - which are task-related

Threats/Power

TH Threat - action if others do not comply

PW Power - one's ability to dominate others

\section{$\underline{\text { Reactions }}$}

RP Positive

RN Negative

\section{$\underline{\text { Procedural comments }}$}

P1 Discuss one issue - deal with one issue at a time

PC Compromise - suggest compromise or willingness to concede

PP Package/Trade-off - involving two or more issues

PM Moving on - without resolution

PV Vote on one issue - before moving on to the next

PX Reciprocity - concession in exchange for future concession

PO Other - procedure for managing the discussion

PT Time - time checks

$\underline{\text { Miscellaneous/Other }}$

CS Creative solutions - potential solutions outside boundaries of task

MI Miscellaneous - general on-task related statements and comments

OT Off-task questions, answers, and/or comments 
Section C: Sample sequences of integrative negotiation

Reciprocal

Negotiator A: I was thinking of going towards $70 \%$ for floor space and then like 19 degrees for temperature?

Negotiator B: I'm ok with $70 \%$ but could we make it 21 degrees?

Negotiator A: $60 \%$ and 21 degrees?

Complementary

Negotiator A: how about you? What's your most important issue?

Negotiator B: probably the grand opening date?

Cooperative relationship management

Negotiator A: I'd like to cooperate with you about the new grocery store.

Negotiator B: yes, I look forward to working with you in our new store. 